\title{
PERMANENCE UNDER STRONG AGGRESSIONS IS POSSIBLE
}

\section{LA PERMANENCE D'ESPÈCES QUI S'ENTRE-DÉVORENT EST POSSIBLE}

\author{
Santiago CANO-CASANOVA ${ }^{\mathrm{a}}$, Julián LÓPEZ-GÓMEZ ${ }^{\mathrm{b}, *}$ \\ ${ }^{a}$ Departamento de Matemática Aplicada y Computación, Universidad Pontificia Comillas de Madrid, \\ 28015-Madrid, Spain \\ ${ }^{\mathrm{b}}$ Departamento de Matemática Aplicada, Universidad Complutense de Madrid, 28040-Madrid, Spain
}

Received 27 February 2002, accepted 18 November 2002

\begin{abstract}
This paper analyzes the limiting behavior of the positive solutions of a general class of sublinear elliptic weighted mixed boundary value problems as the amplitude of the positive part of the lower order terms of the differential operator blows up to infinity. The main result establishes that the positive solutions approximate zero within the support of the positive part of the potential, whereas they stabilize to the positive solution of a certain elliptic mixed boundary value problem on its complement. Further, we use this result for deriving some general principles in competing species dynamics. Precisely, we shall show that in the presence of a refuge region two competing species must coexist if their reproduction rates are sufficiently large, independently of the strength of the competition. It should be emphasized that the abstract theory developed here allows measuring how large the reproduction rates should be for being permanent, providing us, simultaneously, with the limiting behavior of each of the species separately. Basically, when the pressure from the competitor grows the tackled species concentrates within its refuge. The results of this paper are substantial extensions of some pioneer results found by one of the authors in [16, Section 4]. The main ingredients in deriving the main results of this paper are the continuous dependence of the principal eigenvalue with respect to a general class of perturbations of the domain around its Dirichlet boundary - very recent result coming from [6] - and the continuous dependence of the positive solutions of the sublinear problem - coming from [7].

(C) 2003 L'Association Publications de l'Institut Henri Poincaré. Published by Elsevier B.V. All rights reserved
\end{abstract}

MSC: 35B $25 ; 35 \mathrm{~J} 25 ; 35 \mathrm{~K} 57$

RÉSUMÉ. - On étudie le comportement asymptotique des solutions positives d'une classe très générale de problèmes aux limites non linéaires elliptiques lorsque l'amplitude du potentiel

\footnotetext{
* Corresponding author.

E-mail addresses: scano@dmc.icai.upco.es (S. Cano-Casanova), lopez_gomez@mat.ucm.es (J. López-Gómez).
} 
d'ordre zéro de l'opérateur différentiel tend vers l'infini. En particulier, on verra que la solution tend vers zéro sur le support de la partie positive du potentiel, tandis qu'elle converge vers la solution positive d'un certain problème aux limites elliptiques auxiliaires sur la région ou le potentiel est nul. De plus, on va tirer de ce résultat de convergence un postulat biologique concernant la lutte pour la vie des espèces qui s'entredévorent : indépendamment de l'intensité des agressions, en présence d'un refuge pour chaque compétiteur les espèces coexistent si leurs coefficients d'accroissement sont assez longs. Il faut préciser qu'avec la théorie développée ici on peut mesurer le coefficient d'accroissement critique des espèces pour avoir la coexistence. Naturellement, l'espèce agressée va se concentrer sur les refuges correspondants si l'intensité des agressions croît. Tous les résultats obtenus ici sont des généralisations substantielles des résultats [16, Section 4]. Pour démontrer ces résultats on utilise la dépendance continue de la première valeur propre, et de la solution positive même du problème aux limites non linéaire, par rapport aux perturbations du domaine (cf. [6] et [7]).

(c) 2003 L'Association Publications de l'Institut Henri Poincaré. Published by Elsevier B.V. All rights reserved

\section{Introduction}

In this paper we analyze the limiting behavior as $\gamma \nearrow \infty$ of the positive solutions of the following elliptic boundary value problem

$$
\begin{cases}\mathfrak{L} u+\gamma V(x) u=\lambda W(x) u-\mathcal{X}(x) f(x, u) u & \text { in } \Omega, \\ \mathfrak{B}(b) u=0 & \text { on } \partial \Omega,\end{cases}
$$

where $\mathcal{X}, V, W \in L_{\infty}(\Omega), \mathcal{X}$ and $V$ belong to a certain class of nonnegative potentials to be introduced later, and we assume the following:

(a) $\Omega$ is a bounded domain of $\mathbb{R}^{N}, N \geqslant 1$, of class $\mathcal{C}^{2}$, i.e., $\bar{\Omega}$ is an $N$-dimensional compact connected $\mathcal{C}^{2}$-submanifold of $\mathbb{R}^{N}$ with boundary $\partial \Omega$ of class $\mathcal{C}^{2}$.

(b) $\gamma, \lambda \in \mathbb{R}$, and

$$
\mathfrak{L}:=-\sum_{i, j=1}^{N} \alpha_{i j}(x) \frac{\partial^{2}}{\partial x_{i} \partial x_{j}}+\sum_{i=1}^{N} \alpha_{i}(x) \frac{\partial}{\partial x_{i}}+\alpha_{0}(x)
$$

is an uniformly strongly elliptic second order differential operator in $\Omega$ with

$$
\alpha_{i j}=\alpha_{j i} \in \mathcal{C}^{1}(\bar{\Omega}), \quad \alpha_{i} \in \mathcal{C}(\bar{\Omega}), \quad \alpha_{0} \in L_{\infty}(\Omega), \quad 1 \leqslant i, j \leqslant N .
$$

Subsequently, we denote by $\mu>0$ the ellipticity constant of $\mathfrak{L}$ in $\Omega$. Then, for any $\xi \in \mathbb{R}^{N} \backslash\{0\}$ and $x \in \bar{\Omega}$ we have that

$$
\sum_{i, j=1}^{N} \alpha_{i j}(x) \xi_{i} \xi_{j} \geqslant \mu|\xi|^{2}
$$

(c) $\mathfrak{B}(b)$ stands for the boundary operator

$$
\mathfrak{B}(b) u:= \begin{cases}u & \text { on } \Gamma_{0}, \\ \partial_{\nu} u+b u & \text { on } \Gamma_{1},\end{cases}
$$


S. CANO-CASANOVA, J. LÓPEZ-GÓMEZ / Ann. I. H. Poincaré - AN 20 (2003) 999-1041 1001

where $\Gamma_{0}$ and $\Gamma_{1}$ are two disjoint open and closed subsets of $\partial \Omega$ with

$$
\Gamma_{0} \cup \Gamma_{1}=\partial \Omega,
$$

$b \in \mathcal{C}\left(\Gamma_{1}\right)$, and

$$
v=\left(v_{1}, \ldots, v_{N}\right) \in \mathcal{C}^{1}\left(\Gamma_{1} ; \mathbb{R}^{N}\right)
$$

is an outward pointing nowhere tangent vector field. Necessarily, $\Gamma_{0}$ and $\Gamma_{1}$ possess finitely many components. Note that, $\mathfrak{B}(b)$ is the Dirichlet boundary operator on $\Gamma_{0}$, denoted in the sequel by $\mathfrak{D}$, and the Neumann or a first order regular oblique derivative boundary operator on $\Gamma_{1}$. It should be pointed out that either $\Gamma_{0}$ or $\Gamma_{1}$ might be empty.

(d) The function $f: \bar{\Omega} \times[0, \infty) \rightarrow \mathbb{R}$ satisfies

$$
f \in \mathcal{C}^{1}(\bar{\Omega} \times[0, \infty) ; \mathbb{R}), \quad \lim _{u \nearrow \infty} f(x, u)=\infty \quad \text { uniformly in } \bar{\Omega},
$$

and

$$
\partial_{u} f(\cdot, u)>0 \quad \text { for all } u \geqslant 0 .
$$

It should be noted that

$$
f(\cdot, 0) \in \mathcal{C}^{1}(\bar{\Omega} ; \mathbb{R})
$$

and that there is no sign restriction on $f(\cdot, 0)$ in $\Omega$. Moreover, (1.2) implies

$$
f(\cdot, 0)=\inf _{\xi>0} f(\cdot, \xi)
$$

As far as the weight functions $\mathcal{X}, V \in L_{\infty}(\Omega)$ are concerned, it is assumed that

$$
\mathcal{X}, V \in \mathcal{A}_{\Gamma_{0}, \Gamma_{1}}(\Omega),
$$

where $\mathcal{A}_{\Gamma_{0}, \Gamma_{1}}(\Omega)$ is the class of nonnegative potentials introduced by the following definition.

Definition 1.1. - Given $a \in L_{\infty}^{+}(\Omega)\left(a \in L_{\infty}(\Omega)\right.$ such that $\left.a \geqslant 0\right)$, it is said that

$$
a \in \mathcal{A}_{\Gamma_{0}, \Gamma_{1}}(\Omega)
$$

if an open subset $\Omega_{a}^{0}$ of $\Omega$ and a compact subset $K_{a}$ of $\bar{\Omega}$ with Lebesgue measure zero exist for which

$$
\begin{aligned}
& K_{a} \cap\left(\bar{\Omega}_{a}^{0} \cup \Gamma_{1}\right)=\emptyset, \\
& \Omega_{a}^{+}:=\{x \in \Omega: a(x)>0\}=\Omega \backslash\left(\bar{\Omega}_{a}^{0} \cup K_{a}\right),
\end{aligned}
$$

and each of the following four conditions is satisfied:

$\left(\mathcal{A}_{1}\right) \Omega_{a}^{0}$ possesses finitely many components of class $\mathcal{C}^{2}$, say $\Omega_{a}^{0, j}, 1 \leqslant j \leqslant m$, such that

$$
\bar{\Omega}_{a}^{0, i} \cap \bar{\Omega}_{a}^{0, j}=\emptyset \quad \text { if } i \neq j
$$


and

$$
\operatorname{dist}\left(\Gamma_{1}, \partial \Omega_{a}^{0} \cap \Omega\right)>0 .
$$

Thus, if we denote by $\Gamma_{1}^{i}, 1 \leqslant i \leqslant n_{1}$, the components of $\Gamma_{1}$, then for each $1 \leqslant i \leqslant n_{1}$ either $\Gamma_{1}^{i} \subset \partial \Omega_{a}^{0}$ or else $\Gamma_{1}^{i} \cap \partial \Omega_{a}^{0}=\emptyset$. Moreover, if $\Gamma_{1}^{i} \subset \partial \Omega_{a}^{0}$, then $\Gamma_{1}^{i}$ must be a component of $\partial \Omega_{a}^{0}$. Indeed, if $\Gamma_{1}^{i} \cap \partial \Omega_{a}^{0} \neq \emptyset$ but $\Gamma_{1}^{i}$ is not a component of $\partial \Omega_{a}^{0}$, then $\operatorname{dist}\left(\Gamma_{1}^{i}, \partial \Omega_{a}^{0} \cap \Omega\right)=0$.

$\left(\mathcal{A}_{2}\right)$ Let $\left\{i_{1}, \ldots, i_{p}\right\}$ denote the subset of $\left\{1, \ldots, n_{1}\right\}$ for which

$$
\Gamma_{1}^{j} \cap \partial \Omega_{a}^{0}=\emptyset \quad \Longleftrightarrow j \in\left\{i_{1}, \ldots, i_{p}\right\} .
$$

Then, $a$ is bounded away from zero in any compact subset of

$$
\Omega_{a}^{+} \cup \bigcup_{j=1}^{p} \Gamma_{1}^{i_{j}} .
$$

Note that if $\Gamma_{1} \subset \partial \Omega_{a}^{0}$, then we are only imposing a to be bounded away from zero in any compact subset of $\Omega_{a}^{+}$.

$\left(\mathcal{A}_{3}\right)$ Let $\Gamma_{0}^{i}, 1 \leqslant i \leqslant n_{0}$, denote the components of $\Gamma_{0}$, and let $\left\{i_{1}, \ldots, i_{q}\right\}$ be the subset of $\left\{1, \ldots, n_{0}\right\}$ for which

$$
\left(\partial \Omega_{a}^{0} \cup K_{a}\right) \cap \Gamma_{0}^{j} \neq \emptyset \quad \Longleftrightarrow j \in\left\{i_{1}, \ldots, i_{q}\right\} .
$$

Then, $a$ is bounded away from zero on any compact subset of

$$
\Omega_{a}^{+} \cup\left[\bigcup_{j=1}^{q} \Gamma_{0}^{i_{j}} \backslash\left(\partial \Omega_{a}^{0} \cup K_{a}\right)\right] .
$$

Note that if $\left(\partial \Omega_{a}^{0} \cup K_{a}\right) \cap \Gamma_{0}=\emptyset$, then we are only imposing that $a$ is bounded away from zero on any compact subset of $\Omega_{a}^{+}$.

$\left(\mathcal{A}_{4}\right)$ For any $\eta>0$ there exist a natural number $\ell_{a}(\eta) \geqslant 1$ and $\ell_{a}(\eta)$ open subsets of $\mathbb{R}^{N}, G_{j}^{\eta}, 1 \leqslant j \leqslant \ell_{a}(\eta)$, with $\left|G_{j}^{\eta}\right|<\eta, 1 \leqslant j \leqslant \ell_{a}(\eta)$, such that

$$
\bar{G}_{i}^{\eta} \cap \bar{G}_{j}^{\eta}=\emptyset \quad \text { if } i \neq j, \quad K_{a} \subset \bigcup_{j=1}^{\ell_{a}(\eta)} G_{j}^{\eta},
$$

and, for each $1 \leqslant j \leqslant \ell_{a}(\eta)$, the open set $G_{j}^{\eta} \cap \Omega$ is connected and of class $\mathcal{C}^{2}$. Subsequently, it will be said that $a \in \mathcal{A}_{\Gamma_{0}, \Gamma_{1}}^{+}(\Omega)$ if $a \in \mathcal{A}_{\Gamma_{0}, \Gamma_{1}}(\Omega)$ and $\Omega_{a}^{0}=\emptyset$.

Remark 1.2. - When $a \in \mathcal{A}_{\Gamma_{0}, \Gamma_{1}}^{+}(\Omega)$, we have that

$$
K_{a} \cap \Gamma_{1}=\emptyset \quad \wedge \quad \Omega_{a}^{+}:=\{x \in \Omega: a(x)>0\}=\Omega \backslash K_{a} .
$$


Moreover, if we denote by $\Gamma_{0}^{i}, 1 \leqslant i \leqslant n_{0}$, the components of $\Gamma_{0}$ and by $\left\{i_{1}, \ldots, i_{q}\right\}$ the subset of $\left\{1, \ldots, n_{0}\right\}$ for which

$$
K_{a} \cap \Gamma_{0}^{j} \neq \emptyset \quad \Longleftrightarrow j \in\left\{i_{1}, \ldots, i_{q}\right\}
$$

then, $\left(\mathcal{A}_{2}\right)$ and $\left(\mathcal{A}_{3}\right)$ are satisfied if, and only if, $a$ is bounded away from zero on compact subsets of

$$
\Omega_{a}^{+} \cup \Gamma_{1} \cup\left(\bigcup_{j=1}^{q} \Gamma_{0}^{i_{j}} \backslash K_{a}\right) .
$$

If, in addition, $K_{a} \cap \Gamma_{0}=\emptyset$, then $\left(\mathcal{A}_{2}\right)$ and $\left(\mathcal{A}_{3}\right)$ are satisfied if, and only if, $a$ is bounded away from zero on compact subsets of $\Omega_{a}^{+} \cup \Gamma_{1}$.

Also, this paper assumes that $\Omega_{V}^{0}$ is connected and

$$
\Gamma_{0}^{0}:=\partial \Omega_{V}^{0} \backslash \Gamma_{1} \subset \Omega, \quad \operatorname{dist}\left(\Gamma_{1}, \Gamma_{0}^{0}\right)>0 .
$$

Note that, since $V \in \mathcal{A}_{\Gamma_{0}, \Gamma_{1}}(\Omega)$, the second relation of (1.7) follows from (1.6). As an immediate consequence from (1.7), for each $1 \leqslant i \leqslant n_{1}$ either $\Gamma_{1}^{i} \subset \partial \Omega_{V}^{0}$ or else $\Gamma_{1}^{i} \cap \partial \Omega_{V}^{0}=\emptyset$. Moreover, $\Gamma_{1}^{i}$ must be a component of $\partial \Omega_{V}^{0}$ if $\Gamma_{1}^{i} \subset \partial \Omega_{V}^{0}$. Assumption (1.7) allow us to apply [7, Theorem 4.2] (cf. Theorem 2.19 of Section 2 here in). Subsequently, for any $\delta \geqslant 0$ sufficiently small, $\Omega_{V}^{\delta}$ will stand for the open set

$$
\Omega_{V}^{\delta}:=\Omega_{V}^{0} \cup\left\{x \in \Omega: \operatorname{dist}\left(x, \Gamma_{0}^{0}\right)<\delta\right\}
$$

and we assume that there is a sequence $v_{n}, n \geqslant 1$, such that $\lim _{n \rightarrow \infty} v_{n}=0$ for which some of the general assumptions (a)-(d) or (e) of Theorem 2.19 of Section 2 with

$$
\left(a, \Omega_{0}, \Omega_{n}\right)=\left(\mathcal{X}, \Omega_{V}^{0}, \Omega_{V}^{v_{n}}\right), \quad n \geqslant 1
$$

are satisfied. Moreover, we also assume that, for each $\delta \geqslant 0$ sufficiently small,

$$
\mathcal{X} \in \mathcal{A}_{\partial \Omega_{V}^{\delta} \backslash \Gamma_{1}, \partial \Omega_{V}^{\delta} \cap \Gamma_{1}}\left(\Omega_{V}^{\delta}\right) .
$$

Throughout this paper, (1.1) will be refereed to as problem $P[\gamma, \lambda, \Omega, \mathfrak{B}(b)]$, and $\Lambda[\gamma, \Omega, \mathfrak{B}(b)]$ will stand for the set of values of $\lambda \in \mathbb{R}$ for which $P[\gamma, \lambda, \Omega, \mathfrak{B}(b)]$ possesses a positive solution. Thanks to the main result of [5], $P[\gamma, \lambda, \Omega, \mathfrak{B}(b)]$ has a unique positive solution if $\lambda \in \Lambda[\gamma, \Omega, \mathfrak{B}(b)]$. Throughout this paper such a solution will be denoted by

$$
u_{[\mathfrak{L}+\gamma V, \lambda W, \mathcal{X}, \Omega, \mathfrak{B}(b)]} \cdot
$$

To state our main result we need to introduce some notation. Given any proper subdomain $\Omega_{0}$ of $\Omega$ of class $\mathcal{C}^{2}$ satisfying

$$
\operatorname{dist}\left(\Gamma_{1}, \partial \Omega_{0} \cap \Omega\right)>0
$$


we shall denote by $\mathfrak{B}\left(b, \Omega_{0}\right)$ the boundary operator defined from $\mathfrak{B}(b)$ through

$$
\mathfrak{B}\left(b, \Omega_{0}\right):= \begin{cases}\mathfrak{D} & \text { on } \partial \Omega_{0} \cap \Omega, \\ \mathfrak{B}(b) & \text { on } \partial \Omega_{0} \cap \partial \Omega .\end{cases}
$$

The main result of this paper reads as follows.

THEOREM 1.3. - Beside all previous general assumptions, suppose the following conditions hold:

(1) $\lambda \in \Lambda\left[0, \Omega_{V}^{0}, \mathfrak{B}\left(b, \Omega_{V}^{0}\right)\right]$,

(2) $\gamma_{0}>0$ exists for which

$$
\lambda \in \bigcap_{\gamma \geqslant \gamma_{0}} \Lambda[\gamma, \Omega, \mathfrak{B}(b)]
$$

(3) For each $1 \leqslant i \leqslant N$,

$$
v_{i}:=\sum_{j=1}^{N} \alpha_{i j} n_{j} \quad \text { on } \Gamma_{1} \cap \partial \Omega_{V}^{0}
$$

Then, for each $p \in[1, \infty)$,

$$
\lim _{\gamma \nearrow \infty}\left\|u_{[\mathfrak{L}+\gamma V, \lambda W, \mathcal{X}, \Omega, \mathfrak{B}(b)]}-u_{\left[\mathfrak{L}, \lambda W, \mathcal{X}, \Omega_{V}^{0}, \mathfrak{B}\left(b, \Omega_{V}^{0}\right)\right]}\right\|_{L_{p}\left(\Omega_{V}^{0}\right)}=0
$$

and

$$
\lim _{\gamma \nearrow \infty}\left\|u_{[\mathfrak{L}+\gamma V, \lambda W, \mathcal{X}, \Omega, \mathfrak{B}(b)]}\right\|_{L_{\infty}(K)}=0
$$

in any compact subset $K$ of $\bar{\Omega} \backslash \bar{\Omega}_{V}^{0}$. In particular,

$$
\lim _{\gamma>\infty} u_{[\mathfrak{L}+\gamma V, \lambda W, \mathcal{X}, \Omega, \mathfrak{B}(b)]}=\left\{\begin{array}{ll}
u_{\left[\mathfrak{L}, \lambda W, \mathcal{X}, \Omega_{V}^{0}, \mathfrak{B}\left(b, \Omega_{V}^{0}\right)\right]} & \text { in } \Omega_{V}^{0}, \\
0 & \text { in } \Omega \backslash \Omega_{V}^{0}
\end{array} \quad \text { a.e. in } \Omega .\right.
$$

This theorem provides us with a substantially sharper version of [16, Theorem 4.1], where a very special case was treated. No other result of this nature seems to available in the mathematical literature. The proof of Theorem 1.3 is based upon the construction of an adequate supersolution of problem $P[\gamma, \lambda, \Omega, \mathfrak{B}(b)]$ for $\gamma>0$ sufficiently large. Such construction is extremely delicate, since it contains a number of very fine technical details. In constructing these supersolutions one should slightly enlarge the vanishing set $\Omega_{V}^{0}$ of the potential $V$ in the domain $\Omega$ and it is in this precise moment when we need to use the results on continuous dependence with respect to the underlying domain of the positive solutions of $P[\gamma, \lambda, \Omega, \mathfrak{B}(b)]$. Those results, coming from [7], will be collected in Section 2.

An outline of this paper is as follows. In Section 2 we fix the main notations and give some previous results - more or less known - that are going to be used throughout this paper. In Section 3 we prove Theorem 1.3. In Section 4 we give some sufficient conditions so that Theorem 1.3 can be applied. Finally, in Section 5 we use Theorem 1.3 
to show that in the presence of refuge areas two competing species will coexist provided their reproduction rates are sufficiently large. How large those rates must be will be ascertained in terms of the principal eigenvalues of some elliptic operators supported in the refuges of the species. Quite strikingly, the critical reproduction rates are independent of the aggression caused by competition. Further, it will be shown that as soon as the competition level grows the corresponding species must concentrate in its refuge area. Actually, they must segregate toward their respective refuges as the "amplitude" of the competitive interaction becomes large. So, in the presence of a refuge, the stress caused by competition forces the species to concentrate in its refuge area. In obtaining all those sort of biological principles we will use a general class of Lotka-Volterra competing species models with diffusion and transports effects.

Competition, as most ecologists employ the word, means the active demand by a number of individuals of the same species - intraspecific competition - or members of a number of species at the same trophic level - interspecific competition - for a common resource or requirement that is actually, or potentially, limiting, [4,27]. It is commonly agreed that this definition is consistent with the assumptions of the LotkaVolterra equations, which still seems to conform the basis of the mathematical theory of competition. So, our results might have a significant value from the point of view of mathematical biology. Actually, the model is providing us with an idealized behavior, apparently described for the first time, against which reality can be judged and measured.

The weakest part of those models from the modeling perspective is the diffusion term. Nevertheless, although filled with hard to justify (or even doubtful) hypothesis, the competition Lotka-Volterra model does not suffer so much faults from the point of view of population dynamics.

It should be noted that the concentration principle described in Section 5 cannot occur in homogeneous models, but exclusively in heterogeneous ones. The mathematical difficulties that one must overcome to deal with degenerate spatially heterogeneous problems might explain the lack of mathematical results in that direction (cf., e.g., $[20,22]$, and the references there in).

\section{Preliminaries, notations and previous results}

This section fixes some notations and collects some of the main results of $[1,3,5,6]$ and [7]; those results will be used in subsequent sections.

For each $p>1$ we consider

$$
\begin{aligned}
& W_{p, \mathfrak{B}(b)}^{2}(\Omega):=\left\{u \in W_{p}^{2}(\Omega): \mathfrak{B}(b) u=0\right\}, \\
& W_{\mathfrak{B}(b)}^{2}(\Omega):=\bigcap_{p>1} W_{p, \mathfrak{B}(b)}^{2}(\Omega) \subset H^{2}(\Omega),
\end{aligned}
$$

and use the natural product order in $L_{p}(\Omega) \times L_{p}(\partial \Omega)$,

$$
\left(f_{1}, g_{1}\right) \geqslant\left(f_{2}, g_{2}\right) \quad \Longleftrightarrow \quad f_{1} \geqslant f_{2} \wedge g_{1} \geqslant g_{2} .
$$

It will be said that $\left(f_{1}, g_{1}\right)>\left(f_{2}, g_{2}\right)$ if $\left(f_{1}, g_{1}\right) \geqslant\left(f_{2}, g_{2}\right)$ and $\left(f_{1}, g_{1}\right) \neq\left(f_{2}, g_{2}\right)$. 
Since $b \in \mathcal{C}\left(\Gamma_{1}\right)$, it follows from [21] that, for each $p>1$,

$$
\mathfrak{B}(b) \in \mathcal{L}\left(W_{p}^{2}(\Omega) ; W_{p}^{2-1 / p}\left(\Gamma_{0}\right) \times W_{p}^{1-1 / p}\left(\Gamma_{1}\right)\right) .
$$

Moreover, for any $\mathcal{P} \in L_{\infty}(\Omega)$ the linear eigenvalue problem

$$
\begin{cases}(\mathfrak{L}+\mathcal{P}) \varphi=\lambda \varphi & \text { in } \Omega \\ \mathfrak{B}(b) \varphi=0 & \text { on } \partial \Omega\end{cases}
$$

possesses a least real eigenvalue, denoted in the sequel by $\sigma[\mathfrak{L}+\mathcal{P}, \Omega, \mathfrak{B}(b)]$ and called the principal eigenvalue of $(\mathfrak{L}+\mathcal{P}, \Omega, \mathfrak{B}(b))$. The principal eigenvalue is simple and associated with it there is a positive eigenfunction, unique up to multiplicative constants; this eigenfunction is called the principal eigenfunction of $(\mathfrak{L}+\mathcal{P}, \Omega, \mathfrak{B}(b))$. Thanks to [1, Theorem 12.1], the principal eigenfunction, subsequently denoted by $\varphi$, satisfies

$$
\varphi \in W_{\mathfrak{B}(b)}^{2}(\Omega) \subset H^{2}(\Omega)
$$

and it is strongly positive in $\Omega$ in the sense that $\varphi(x)>0$ for each $x \in \Omega \cup \Gamma_{1}$ and $\partial_{\nu} \varphi(x)<0$ for each $x \in \Gamma_{0}$. Moreover, $\sigma[\mathfrak{L}+\mathcal{P}, \Omega, \mathfrak{B}(b)]$ is the unique eigenvalue of (2.1) possessing a positive eigenfunction, and it is dominant in the sense that

$$
\operatorname{Re} \sigma>\sigma[\mathfrak{L}+\mathcal{P}, \Omega, \mathfrak{B}(b)]
$$

for any other eigenvalue $\sigma$ of (2.1). Furthermore, setting

$$
(\mathfrak{L}+\mathcal{P})_{p}:=\left.(\mathfrak{L}+\mathcal{P})\right|_{W_{p, \mathfrak{B}(b)}^{2}(\Omega)},
$$

we have that, for each $\omega>-\sigma[\mathfrak{L}+\mathcal{P}, \Omega, \mathfrak{B}(b)]$ and $p>N$, the operator

$$
\left[\omega+(\mathfrak{L}+\mathcal{P})_{p}\right]^{-1} \in \mathcal{L}\left(L_{p}(\Omega)\right)
$$

is a positive, compact and irreducible (cf. [23, V.7.7]).

Throughout this paper, given any proper subdomain $\Omega_{0}$ of $\Omega$ of class $\mathcal{C}^{2}$ with

$$
\operatorname{dist}\left(\Gamma_{1}, \partial \Omega_{0} \cap \Omega\right)>0,
$$

we shall denote by $\mathfrak{B}\left(b, \Omega_{0}\right)$ the boundary operator defined from $\mathfrak{B}(b)$ through

$$
\mathfrak{B}\left(b, \Omega_{0}\right):= \begin{cases}\mathfrak{D} & \text { on } \partial \Omega_{0} \cap \Omega, \\ \mathfrak{B}(b) & \text { on } \partial \Omega_{0} \cap \partial \Omega .\end{cases}
$$

When $\Omega_{0}=\Omega$ we set

$$
\mathfrak{B}(b, \Omega):=\mathfrak{B}(b) .
$$

It should be noted that if $\bar{\Omega}_{0} \subset \Omega$, then $\partial \Omega_{0} \subset \Omega$ and, hence, $\mathfrak{B}\left(b, \Omega_{0}\right)=\mathfrak{D}$, by definition. Also, we will denote by $\sigma\left[\mathfrak{L}+\mathcal{P}, \Omega_{0}, \mathfrak{B}\left(b, \Omega_{0}\right)\right]$ the principal eigenvalue 
of the linear boundary value problem

$$
\begin{cases}(\mathfrak{L}+\mathcal{P}) \psi=\lambda \psi & \text { in } \Omega_{0} \\ \mathfrak{B}\left(b, \Omega_{0}\right) \psi=0 & \text { on } \partial \Omega_{0}\end{cases}
$$

We now recall the concept of principal eigenvalue for a domain with several components.

DEFINITION 2.1. - Suppose $\Omega_{0}$ is an open subset of $\Omega$ with a finite number of components of class $\mathcal{C}^{2}$, say $\Omega_{0}^{j}, 1 \leqslant j \leqslant m$, such that $\bar{\Omega}_{0}^{i} \cap \bar{\Omega}_{0}^{j}=\emptyset$ if $i \neq j$ and

$$
\operatorname{dist}\left(\Gamma_{1}, \partial \Omega_{0} \cap \Omega\right)>0 .
$$

Then, the principal eigenvalue of $\left(\mathfrak{L}+\mathcal{P}, \Omega_{0}, \mathfrak{B}\left(b, \Omega_{0}\right)\right)$ is defined through

$$
\sigma\left[\mathfrak{L}+\mathcal{P}, \Omega_{0}, \mathfrak{B}\left(b, \Omega_{0}\right)\right]:=\min _{1 \leqslant j \leqslant m} \sigma\left[\mathfrak{L}+\mathcal{P}, \Omega_{0}^{j}, \mathfrak{B}\left(b, \Omega_{0}^{j}\right)\right] .
$$

Remark 2.2. - Since $\Omega_{0}$ is of class $\mathcal{C}^{2}$, it follows from (2.5) that each of the principal eigenvalues $\sigma\left[\mathfrak{L}+\mathcal{P}, \Omega_{0}^{j}, \mathfrak{B}\left(b, \Omega_{0}^{j}\right)\right], \quad 1 \leqslant j \leqslant m$, is well defined. This shows the consistency of Definition 2.1.

Suppose $p>N$ and $\mathcal{P} \in L_{\infty}(\Omega)$. Then, a function $\bar{u} \in W_{p}^{2}(\Omega)$ is said to be a positive strict supersolution of $(\mathfrak{L}+\mathcal{P}, \Omega, \mathfrak{B}(b))$ if

$$
\bar{u} \geqslant 0 \wedge((\mathfrak{L}+\mathcal{P}) \bar{u}, \mathfrak{B}(b) \bar{u})>0 .
$$

A function $u \in W_{p}^{2}(\Omega)$ is said to be strongly positive if $u(x)>0$ for each $x \in \Omega \cup \Gamma_{1}$ and $\partial_{\beta} u(x)<0$ for each $x \in \Gamma_{0}$ satisfying $u(x)=0$ and any outward pointing nowhere tangent vector field $\beta \in \mathcal{C}^{1}\left(\Gamma_{0} ; \mathbb{R}^{N}\right)$. Finally, $(\mathfrak{L}+\mathcal{P}, \Omega, \mathfrak{B}(b))$ is said to satisfy the strong maximum principle if $p>N, u \in W_{p}^{2}(\Omega)$, and $((\mathfrak{L}+\mathcal{P}) u, \mathfrak{B}(b) u)>0$ imply that $u$ is strongly positive. It should be recalled that for any $p>N$

$$
W_{p}^{2}(\Omega) \hookrightarrow \mathcal{C}^{2-N / p}(\bar{\Omega})
$$

and that any function $u \in W_{p}^{2}(\Omega)$ is a.e. in $\Omega$ twice differentiable (cf., e.g., [25, Theorem VIII.1]).

The following characterization of the strong maximum principle provides us with one of the main technical tools to make most of the comparisons of this paper. It goes back to $[17,18]$, thought the version given here comes from [3].

THEOREM 2.3. - For any $\mathcal{P} \in L_{\infty}(\Omega)$ the following assertions are equivalent:

- $\sigma[\mathfrak{L}+\mathcal{P}, \Omega, \mathfrak{B}(b)]>0$

- $(\mathfrak{L}+\mathcal{P}, \Omega, \mathfrak{B}(b))$ possesses a positive strict supersolution;

- $(\mathfrak{L}+\mathcal{P}, \Omega, \mathfrak{B}(b))$ satisfies the strong maximum principle.

Now, we collect some of the main properties of $\sigma[\mathfrak{L}+\mathcal{P}, \Omega, \mathfrak{B}(b)]$; they are taken from [6, Proposition 3.2, 3.3]. 
1008 S. CANO-CASANOVA, J. LÓPEZ-GÓMEZ / Ann. I. H. Poincaré - AN 20 (2003) 999-1041

PROPOSITION 2.4. - Let $\Omega_{0}$ be a proper subdomain of $\Omega$ of class $\mathcal{C}^{2}$ satisfying (2.2). Then,

$$
\sigma[\mathfrak{L}+\mathcal{P}, \Omega, \mathfrak{B}(b)]<\sigma\left[\mathfrak{L}+\mathcal{P}, \Omega_{0}, \mathfrak{B}\left(b, \Omega_{0}\right)\right],
$$

where $\mathfrak{B}\left(b, \Omega_{0}\right)$ is the boundary operator defined by (2.3).

Proposition 2.5. - Let $\mathcal{P}_{1}, \mathcal{P}_{2} \in L_{\infty}(\Omega)$ such that $\mathcal{P}_{1}<\mathcal{P}_{2}$ in a set of positive Lebesgue measure. Then,

$$
\sigma\left[\mathfrak{L}+\mathcal{P}_{1}, \Omega, \mathfrak{B}(b)\right]<\sigma\left[\mathfrak{L}+\mathcal{P}_{2}, \Omega, \mathfrak{B}(b)\right] .
$$

A crucial result for the mathematical analysis carried out in the next sections is the continuous dependence of the principal eigenvalue $\sigma[\mathfrak{L}+\mathcal{P}, \Omega, \mathfrak{B}(b)]$ with respect to the perturbations of the domain around its Dirichlet boundary. To state it we need introducing the following concepts.

DEFINITION 2.6. - Let $\Omega_{0}$ be a bounded domain of $\mathbb{R}^{N}$ with boundary $\partial \Omega_{0}=$ $\Gamma_{0}^{0} \cup \Gamma_{1}$ such that $\Gamma_{0}^{0} \cap \Gamma_{1}=\emptyset$, where $\Gamma_{0}^{0}$ satisfies the same requirements as $\Gamma_{0}$, and $\Omega_{n}, n \geqslant 1$, a sequence of bounded domains of $\mathbb{R}^{N}$ with boundaries $\partial \Omega_{n}=\Gamma_{0}^{n} \cup \Gamma_{1}$ of class $\mathcal{C}^{2}$ such that

$$
\Gamma_{0}^{n} \cap \Gamma_{1}=\emptyset, \quad n \geqslant 1,
$$

and $\Gamma_{0}^{n}, n \geqslant 1$, satisfies the same requirements as $\Gamma_{0}$. Then, it is said that $\Omega_{n}$ converges to $\Omega_{0}$ from the exterior if, for each $n \geqslant 1$,

$$
\Omega_{0} \subset \Omega_{n+1} \subset \Omega_{n} \text { and } \bigcap_{n=1}^{\infty} \bar{\Omega}_{n}=\bar{\Omega}_{0}
$$

Throughout the remaining of this paper it is said that $v=\left(v_{1}, \ldots, v_{N}\right)$ is the conormal vector field if

$$
v_{i}:=\sum_{j=1}^{N} \alpha_{i j} n_{j}, \quad 1 \leqslant i \leqslant N,
$$

where $n=\left(n_{1}, \ldots, n_{N}\right)$ is the outward unit normal to $\Omega$ on $\Gamma_{1}$. In this case $\partial_{v}$ will be called the conormal derivative. Let $\mu>0$ denote the ellipticity constant of $\mathfrak{L}$ and assume that (2.8) is satisfied. Then,

$$
\langle v, n\rangle=\sum_{i, j=1}^{N} \alpha_{i j} n_{j} n_{i} \geqslant \mu|n|^{2}=\mu>0
$$

and, therefore, $v$ is an outward pointing nowhere tangent vector field. It should be noted that $v \in \mathcal{C}^{1}\left(\Gamma_{1} ; \mathbb{R}^{N}\right)$, since $\alpha_{i j} \in \mathcal{C}^{1}(\bar{\Omega}), 1 \leqslant i, j \leqslant N$, and $\Gamma_{1}$ is of class $\mathcal{C}^{2}$. It is time for establishing the main result about the continuous dependence of the principal eigenvalue with respect to the perturbations of the domain around its Dirichlet boundary; it goes back to [6, Theorem 7.1]. 
Theorem 2.7 (Exterior Continuous Dependence). - Suppose (2.8) and consider $\mathcal{P} \in L_{\infty}(\Omega)$. Let $\Omega_{0}$ be a proper subdomain of $\Omega$ with boundary of class $\mathcal{C}^{2}$ such that

$$
\partial \Omega_{0}=\Gamma_{0}^{0} \cup \Gamma_{1}, \quad \Gamma_{0}^{0} \cap \Gamma_{1}=\emptyset,
$$

where $\Gamma_{0}^{0}$ satisfies the same requirements as $\Gamma_{0}$, and let $\Omega_{n} \subset \Omega, n \geqslant 1$, be a sequence of bounded domains of $\mathbb{R}^{N}$ of class $\mathcal{C}^{2}$ converging to $\Omega_{0}$ from the exterior. For each $n \geqslant 0$, let $\mathfrak{B}_{n}(b)$ denote the boundary operator defined through

$$
\mathfrak{B}_{n}(b) u:= \begin{cases}u & \text { on } \Gamma_{0}^{n}, \\ \partial_{\nu} u+b u & \text { on } \Gamma_{1},\end{cases}
$$

where

$$
\Gamma_{0}^{n}:=\partial \Omega_{n} \backslash \Gamma_{1}, \quad n \geqslant 0,
$$

and denote by $\left(\sigma\left[\mathfrak{L}+\mathcal{P}, \Omega_{n}, \mathfrak{B}_{n}(b)\right], \varphi_{n}\right)$ the principal eigen-pair associated with $\left(\mathfrak{L}+\mathcal{P}, \Omega_{n}, \mathfrak{B}_{n}(b)\right)$, where the principal eigenfunction $\varphi_{n}$ is assumed to be normalized so that

$$
\left\|\varphi_{n}\right\|_{H^{1}\left(\Omega_{n}\right)}=1, \quad n \geqslant 0 .
$$

Then, $\varphi_{0} \in W_{\mathfrak{B}_{0}(b)}^{2}\left(\Omega_{0}\right)$ and

$\lim _{n \rightarrow \infty} \sigma\left[\mathfrak{L}+\mathcal{P}, \Omega_{n}, \mathfrak{B}_{n}(b)\right]=\sigma\left[\mathfrak{L}+\mathcal{P}, \Omega_{0}, \mathfrak{B}_{0}(b)\right], \quad \lim _{n \rightarrow \infty}\left\|\left.\varphi_{n}\right|_{\Omega_{0}}-\varphi_{0}\right\|_{H^{1}\left(\Omega_{0}\right)}=0$.

The following result establishes that $(\mathfrak{L}+\mathcal{P}, \Omega, \mathfrak{D})$ satisfies the strong maximum principle if $|\Omega|$ is sufficiently small. It goes back to [17, Theorem 5.1] and [6, Theorem 10.1]. Hereafter, $|\cdot|$ will stand for the Lebesgue measure in $\mathbb{R}^{N}$.

THEOREM 2.8. - Suppose $\mathcal{P} \in L_{\infty}(\Omega)$ and

$$
\alpha_{i j} \in \mathcal{C}(\bar{\Omega}) \cap W_{\infty}^{1}(\Omega), \quad 1 \leqslant i, j \leqslant N .
$$

Then

$$
\liminf _{|\Omega| \searrow 0} \sigma[\mathfrak{L}+\mathcal{P}, \Omega, \mathfrak{D}]|\Omega|^{2 / N} \geqslant \mu \Sigma_{1}\left|B_{1}\right|^{2 / N}
$$

where

$$
B_{1}:=\left\{x \in \mathbb{R}^{N}:|x|<1\right\}, \quad \Sigma_{1}:=\sigma\left[-\Delta, B_{1}, \mathfrak{D}\right],
$$

and $\mu>0$ is the ellipticity constant of $\mathfrak{L}$ in $\Omega$.

Another fundamental result for the mathematical analysis carried out in the subsequent sections is the next one; it goes back to [17, Theorem 6.2] and [6, Theorem 11.4].

THEOREM 2.9. - Assume that (2.8) is satisfied on $\Gamma_{1} \cap \partial \Omega_{V}^{0}$. Then

$$
\lim _{\gamma>\infty} \sigma[\mathfrak{L}+\gamma V, \Omega, \mathfrak{B}(b)]=\sigma\left[\mathfrak{L}, \Omega_{V}^{0}, \mathfrak{B}\left(b, \Omega_{V}^{0}\right)\right] .
$$


The proof of this result can be easily adapted to show that in the particular case when $\Omega_{V}^{0}=\emptyset$ the next theorem follows; it should be noted that condition (2.8) is not required and that the regularity of the coefficients of $\mathfrak{L}$ is weaker than the regularity required in Theorem 2.9.

THEOREM 2.10. - Suppose (2.9) and $V \in \mathcal{A}_{\Gamma_{0}, \Gamma_{1}}^{+}(\Omega)$. Then

$$
\lim _{\gamma \nearrow \infty} \sigma[\mathfrak{L}+\gamma V, \Omega, \mathfrak{B}(b)]=\infty .
$$

Now, we shall state the concept of strong solution for problem $P[\gamma, \lambda, \Omega, \mathfrak{B}(b)]$ and collect the results of [5] that characterize the existence of positive solutions. A function $u$ is said to be a strong solution of $P[\gamma, \lambda, \Omega, \mathfrak{B}(b)]$ if $u \in W_{p}^{2}(\Omega)$ for some $p>N$ and it satisfies (1.1). A function $u$ is said to be a positive solution of $P[\gamma, \lambda, \Omega, \mathfrak{B}(b)]$ if it is a strong solution and $u>0$ in $\Omega$. The solutions of $P[\gamma, \lambda, \Omega, \mathfrak{B}(b)]$ will be regarded as couples $(\lambda, u)$. Accordingly, it will be said that $\left(\lambda_{0}, u_{0}\right)$ is a solution of (1.1) if $u_{0}$ is a solution of $P\left[\gamma, \lambda_{0}, \Omega, \mathfrak{B}(b)\right]$. The following result is [7, Lemma 2.12].

Lemma 2.11. - Suppose $(\lambda, u)$ is a positive solution of (1.1). Then, $u$ is strongly positive in $\Omega$ and $u \in W_{\mathfrak{B}(b)}^{2}(\Omega)$. Moreover,

$$
\sigma[\mathfrak{L}+\gamma V-\lambda W+\mathcal{X} f(\cdot, u), \Omega, \mathfrak{B}(b)]=0 .
$$

In particular, $u \in \mathcal{C}^{1, \vartheta}(\bar{\Omega})$ for each $\vartheta \in(0,1)$, and it is a.e. in $\Omega$ twice differentiable.

The following result characterizes the existence of positive solutions for (1.1); it goes back to [5, Theorem 4.2].

TheOREM 2.12. - Suppose (2.8) on $\Gamma_{1} \cap \partial \Omega_{\mathcal{X}}^{0}$. Then, $P[\gamma, \lambda, \Omega, \mathfrak{B}(b)]$ possesses a positive solution if, and only if,

$$
\sigma[\mathfrak{L}+\gamma V+\mathcal{X} f(\cdot, 0)-\lambda W, \Omega, \mathfrak{B}(b)]<0<\sigma\left[\mathfrak{L}+\gamma V-\lambda W, \Omega_{\mathcal{X}}^{0}, \mathfrak{B}\left(b, \Omega_{\mathcal{X}}^{0}\right)\right] .
$$

Moreover, the positive solution is unique if it exists. Subsequently, it will be denoted by

$$
u_{[\mathfrak{L}+\gamma V, \lambda W, \mathcal{X}, \Omega, \mathfrak{B}(b)]} \cdot
$$

Furthermore, for any $u_{0} \in L_{p}(\Omega), p>N / 2$, the evolutionary problem

$$
\begin{cases}\frac{\partial u}{\partial t}+(\mathfrak{L}+\gamma V) u=\lambda W u-\mathcal{X} f(\cdot, u) u & \text { in } \Omega \times(0, \infty), \\ \mathfrak{B}(b) u=0 & \text { on } \partial \Omega \times(0, \infty), \\ u(\cdot, 0)=u_{0} & \text { in } \Omega\end{cases}
$$

possesses a unique strong solution and, if we denote it by $\Phi_{[\mathfrak{L}+\gamma V, \lambda W, \mathcal{X}, \Omega, \mathfrak{B}(b)]}\left(x, t ; u_{0}\right)$, one has that

$$
\lim _{t \nearrow \infty}\left\|\Phi_{[\mathfrak{L}+\gamma V, \lambda W, \mathcal{X}, \Omega, \mathfrak{B}(b)]}\left(\cdot, t ; u_{0}\right)-u_{[\mathfrak{L}+\gamma V, \lambda W, \mathcal{X}, \Omega, \mathfrak{B}(b)]}\right\|_{C_{0}^{1}(\bar{\Omega})}=0 .
$$

Remark 2.13. - Imposing (2.8) on $\Gamma_{1} \cap \partial \Omega_{\mathcal{X}}^{0}$ is not needed for the uniqueness. 
Arguing as in the proof of Theorem 2.12 (cf. [5, Theorem 4.2]) the following result is easily obtained.

THEOREM 2.14. - Suppose $\mathcal{X} \in \mathcal{A}_{\Gamma_{0}, \Gamma_{1}}^{+}(\Omega)$. Then, $P[\gamma, \lambda, \Omega, \mathfrak{B}(b)]$ has a positive solution if, and only if,

$$
\sigma[\mathfrak{L}+\gamma V-\lambda W+\mathcal{X} f(\cdot, 0), \Omega, \mathfrak{B}(b)]<0 .
$$

Moreover, the positive solution is unique if it exists; subsequently denoted by

$$
u_{[\mathfrak{L}+\gamma V, \lambda W, \mathcal{X}, \Omega, \mathfrak{B}(b)]} \cdot
$$

Furthermore, for any $u_{0} \in L_{p}(\Omega), p>\frac{N}{2}$, the evolutionary problem (2.12) possesses a unique strong solution and, if we denote it by $\Phi_{[\mathfrak{L}+\gamma V, \lambda W, \mathcal{X}, \Omega, \mathfrak{B}(b)]}\left(x, t ; u_{0}\right)$,

$$
\lim _{t>\infty}\left\|\Phi_{[\mathfrak{L}+\gamma V, \lambda W, \mathcal{X}, \Omega, \mathfrak{B}(b)]}\left(\cdot, t ; u_{0}\right)-u_{[\mathfrak{L}+\gamma V, \lambda W, \mathcal{X}, \Omega, \mathfrak{B}(b)]}\right\|_{C_{0}^{1}(\bar{\Omega})}=0 .
$$

In Theorem 2.14 condition (2.8) on $\Gamma_{1} \cap \partial \Omega_{\mathcal{X}}^{0}$ is not required, since $\Omega_{\mathcal{X}}^{0}=\emptyset$. Now, we introduce the concept of positive supersolution.

DEFINITION 2.15. - Given $p>N$, it is said that $u \in W_{p}^{2}(\Omega)$ is a positive supersolution (resp. positive subsolution) of $P[\gamma, \lambda, \Omega, \mathfrak{B}(b)]$ if $u>0$ and

$$
\begin{aligned}
& ([\mathfrak{L}+\gamma V-\lambda W+\mathcal{X} f(\cdot, u)] u, \mathfrak{B}(b) u) \geqslant 0 \\
& (\text { resp. }([\mathfrak{L}+\gamma V-\lambda W+\mathcal{X} f(\cdot, u)] u, \mathfrak{B}(b) u) \leqslant 0) .
\end{aligned}
$$

The following comparison result is crucial in our mathematical analysis; it is [7, Theorem 2.15].

THEOREM 2.16. - Suppose $P[\gamma, \lambda, \Omega, \mathfrak{B}(b)]$ possesses a positive solution, $p>N$, and let $u \in W_{p}^{2}(\Omega)$ be a positive supersolution (resp. subsolution) of $P[\gamma, \lambda, \Omega, \mathfrak{B}(b)]$. Then,

$$
u \geqslant u_{[\mathfrak{L}+\gamma V, \lambda W, \mathcal{X}, \Omega, \mathfrak{B}(b)]} \quad\left(\text { resp. } u \leqslant u_{[\mathfrak{L}+\gamma V, \lambda W, \mathcal{X}, \Omega, \mathfrak{B}(b)]}\right) .
$$

The following results are [7, Theorem 3.1] and [7, Corollary 3.2], respectively. They collect some crucial properties of the families of potentials $\mathcal{A}_{\Gamma_{0}, \Gamma_{1}}(\Omega)$ and $\mathcal{A}_{\Gamma_{0}, \Gamma_{1}}^{+}(\Omega)$. Subsequently, if $a \in \mathcal{A}_{\Gamma_{0}, \Gamma_{1}}(\Omega), \widetilde{\Omega} \subset \Omega$ is an open subset satisfying

$$
\operatorname{dist}(\partial \Omega, \partial \widetilde{\Omega} \cap \Omega)>0
$$

and

$$
a \in \mathcal{A}_{\widetilde{\Gamma}_{0}, \widetilde{\Gamma}_{1}}(\widetilde{\Omega}), \quad \widetilde{\Gamma}_{1}:=\Gamma_{1} \cap \partial \widetilde{\Omega}, \widetilde{\Gamma}_{0}:=\partial \widetilde{\Omega} \backslash \widetilde{\Gamma}_{1},
$$

we will denote by $[\widetilde{\Omega}]_{a}^{0}$ the maximal open subset of $\widetilde{\Omega}$ where the potential $a$ vanishes.

THEOREM 2.17. - Suppose $a \in \mathcal{A}_{\Gamma_{0}, \Gamma_{1}}(\Omega)$ and let $\widetilde{\Omega}$ be an open subdomain of $\Omega$ of class $\mathcal{C}^{2}$ such that

$$
\operatorname{dist}(\partial \Omega, \partial \widetilde{\Omega} \cap \Omega)>0 .
$$


1012 S. CANO-CASANOVA, J. LÓPEZ-GÓMEZ / Ann. I. H. Poincaré - AN 20 (2003) 999-1041

Then, each of the following sets

$$
\widetilde{\Gamma}_{0}:=\partial \widetilde{\Omega} \cap\left(\Gamma_{0} \cup \Omega\right), \quad \widetilde{\Gamma}_{1}:=\partial \widetilde{\Omega} \backslash \widetilde{\Gamma}_{0}=\partial \widetilde{\Omega} \cap \Gamma_{1},
$$

is closed and open in $\partial \widetilde{\Omega}$. Moreover, the following assertions are true:

(a) If $\Omega_{a}^{0} \cap \widetilde{\Omega} \neq \emptyset$ is of class $\mathcal{C}^{2}$ and

$$
\partial \widetilde{\Omega} \cap \Omega \cap \partial\left(\Omega_{a}^{0} \cap \widetilde{\Omega}\right)=\partial \widetilde{\Omega} \cap \Omega \cap \bar{\Omega}_{a}^{0},
$$

then $a \in \mathcal{A}_{\widetilde{\Gamma}_{0}, \widetilde{\Gamma}_{1}}(\widetilde{\Omega})$ and

$$
[\widetilde{\Omega}]_{a}^{0}=\Omega_{a}^{0} \cap \widetilde{\Omega} .
$$

(b) Suppose $\Omega_{a}^{0} \cap \widetilde{\Omega}=\emptyset$ and

$$
\Gamma \cap K_{a} \neq \emptyset \quad \Longrightarrow \quad \Gamma \backslash K_{a} \subset \Omega_{a}^{+}
$$

for any component $\Gamma$ of $\partial \widetilde{\Omega} \cap \Omega$. Then, $a \in \mathcal{A}_{\widetilde{\Gamma}_{0}, \widetilde{\Gamma}_{1}}^{+}(\widetilde{\Omega})$. In particular,

$$
a \in \mathcal{A}_{\Gamma_{0}, \Gamma_{1}}^{+}(\Omega) \quad \Longrightarrow \quad a \in \mathcal{A}_{\widetilde{\Gamma}_{0}, \widetilde{\Gamma}_{1}}^{+}(\widetilde{\Omega}) .
$$

COROLLARY 2.18. - Suppose $a, b \in \mathcal{A}_{\Gamma_{0}, \Gamma_{1}}(\Omega)$ with $\Omega_{b}^{0}$ connected and

$$
\operatorname{dist}\left(\Gamma_{0}, \partial \Omega_{b}^{0} \cap \Omega\right)>0 .
$$

Then,

$$
\widetilde{\Gamma}_{0}:=\partial \Omega_{b}^{0} \cap\left(\Gamma_{0} \cup \Omega\right) \quad \text { and } \quad \widetilde{\Gamma}_{1}:=\partial \Omega_{b}^{0} \backslash \widetilde{\Gamma}_{0}=\partial \Omega_{b}^{0} \cap \Gamma_{1}
$$

are closed and open sets of class $\mathcal{C}^{2}$, and each of the following assertions is true:

(a) If $\Omega_{a}^{0} \cap \Omega_{b}^{0} \neq \emptyset$ is of class $\mathcal{C}^{2}$ and

$$
\partial \Omega_{b}^{0} \cap \Omega \cap \partial\left(\Omega_{a}^{0} \cap \Omega_{b}^{0}\right)=\partial \Omega_{b}^{0} \cap \Omega \cap \bar{\Omega}_{a}^{0},
$$

then $a \in \mathcal{A}_{\widetilde{\Gamma}_{0}, \widetilde{\Gamma}_{1}}\left(\Omega_{b}^{0}\right)$ and

$$
\left[\Omega_{b}^{0}\right]_{a}^{0}=\Omega_{a}^{0} \cap \Omega_{b}^{0}
$$

(b) Suppose $\Omega_{a}^{0} \cap \Omega_{b}^{0}=\emptyset$ and

$$
\Gamma \cap K_{a} \neq \emptyset \quad \Longrightarrow \quad \Gamma \backslash K_{a} \subset \Omega_{a}^{+}
$$

for any component $\Gamma$ of $\partial \Omega_{b}^{0} \cap \Omega$. Then, $a \in \mathcal{A}_{\widetilde{\Gamma}_{0}, \widetilde{\Gamma}_{1}}^{+}\left(\Omega_{b}^{0}\right)$. In particular,

$$
a \in \mathcal{A}_{\Gamma_{0}, \Gamma_{1}}^{+}(\Omega) \Longrightarrow a \in \mathcal{A}_{\widetilde{\Gamma}_{0}, \widetilde{\Gamma}_{1}}^{+}\left(\Omega_{b}^{0}\right) \text {. }
$$

Another crucial result in obtaining Theorem 1.3 is the following theorem; it is [7, Theorem 4.2]. 
S. CANO-CASANOVA, J. LÓPEZ-GÓMEZ / Ann. I. H. Poincaré - AN 20 (2003) 999-1041 1013

THEOREM 2.19. - Suppose $a \in \mathcal{A}_{\Gamma_{0}, \Gamma_{1}}(\Omega)$, let $\Omega_{0}$ be a proper subdomain of $\Omega$ with boundary of class $\mathcal{C}^{2}$ such that

$$
\partial \Omega_{0}=\Gamma_{0}^{0} \cup \Gamma_{1},
$$

where $\Gamma_{0}^{0}$ satisfies the same requirements as $\Gamma_{0}$, and let $\Omega_{n} \subset \Omega, n \geqslant 1$, be a sequence of bounded domains of $\mathbb{R}^{N}$ of class $\mathcal{C}^{2}$ converging to $\Omega_{0}$ from the exterior such that

$$
\operatorname{dist}\left(\partial \Omega, \partial \Omega_{n} \cap \Omega\right)>0, \quad n \geqslant 0 .
$$

For each natural number $n \geqslant 0$ let $\mathfrak{B}_{n}(b)$ be the boundary operator defined by

$$
\mathfrak{B}_{n}(\Omega):= \begin{cases}\mathfrak{D} & \text { on } \Gamma_{0}^{n}, \\ \mathfrak{B}(b) & \text { on } \Gamma_{1},\end{cases}
$$

where

$$
\Gamma_{0}^{n}:=\partial \Omega_{n} \backslash \Gamma_{1}
$$

Then, the following assertions are true:

(a) Suppose (2.8) on $\Gamma_{1} \cap \partial \Omega_{a}^{0}$ and $\emptyset \neq \Omega_{a}^{0} \subset \Omega_{0}$. Then, for each $n \geqslant 0$,

$$
a \in \bigcap_{n=0}^{\infty} \mathcal{A}_{\Gamma_{0}^{n}, \Gamma_{1}}\left(\Omega_{n}\right) \quad \text { and } \quad\left[\Omega_{n}\right]_{a}^{0}=\Omega_{a}^{0}
$$

where $\left[\Omega_{n}\right]_{a}^{0}$ is the corresponding open set of the definition of the class $\mathcal{A}_{\Gamma_{0}^{n}, \Gamma_{1}}\left(\Omega_{n}\right), n \geqslant 0$. Suppose, in addition, that $a=\mathcal{X}$ and $\lambda \in \Lambda\left[\gamma, \Omega_{0}, \mathfrak{B}_{0}(b)\right]$. Then,

$$
\lambda \in \bigcap_{n=0}^{\infty} \Lambda\left[\gamma, \Omega_{n}, \mathfrak{B}_{n}(b)\right]
$$

(b) Suppose $\bar{\Omega}_{0} \cap \bar{\Omega}_{a}^{0}=\emptyset$. Then, $a \in \mathcal{A}_{\Gamma_{0}^{0}, \Gamma_{1}}^{+}\left(\Omega_{0}\right)$. Moreover, $n_{0} \in \mathbb{N}$ exists for which

$$
a \in \bigcap_{n=n_{0}}^{\infty} \mathcal{A}_{\Gamma_{0}^{n}, \Gamma_{1}}^{+}\left(\Omega_{n}\right)
$$

Furthermore,

$$
\lambda \in \bigcap_{n=n_{0}}^{\infty} \Lambda\left[\gamma, \Omega_{n}, \mathfrak{B}_{n}(b)\right]
$$

if $a=\mathcal{X}$ and $\lambda \in \Lambda\left[\gamma, \Omega_{0}, \mathfrak{B}_{0}(b)\right]$.

(c) Suppose $\bar{\Omega}_{a}^{0} \cap \bar{\Omega}_{0} \neq \emptyset, \Omega_{0} \cap \Omega_{a}^{0}=\emptyset$, and $n_{0} \in \mathbb{N}$ exists for which $\Omega_{n} \cap \Omega_{a}^{0}$ is of class $\mathcal{C}^{2}$ and

$$
\partial \Omega_{n} \cap \Omega \cap \partial\left(\Omega_{a}^{0} \cap \Omega_{n}\right)=\partial \Omega_{n} \cap \Omega \cap \bar{\Omega}_{a}^{0}, \quad n \geqslant n_{0} .
$$

Suppose, in addition, that

$$
\Gamma \cap K_{a} \neq \emptyset \quad \Longrightarrow \quad \Gamma \backslash K_{a} \subset \Omega_{a}^{+}
$$


1014 S. CANO-CASANOVA, J. LÓPEZ-GÓMEZ / Ann. I. H. Poincaré - AN 20 (2003) 999-1041

for any component $\Gamma$ of $\Gamma_{0}^{0}$. Then, $a \in \mathcal{A}_{\Gamma_{0}^{0}, \Gamma_{1}}^{+}\left(\Omega_{0}\right)$ and

$$
a \in \bigcap_{n=n_{0}}^{\infty} \mathcal{A}_{\Gamma_{0}^{n}, \Gamma_{1}}\left(\Omega_{n}\right), \quad\left[\Omega_{n}\right]_{a}^{0}=\Omega_{a}^{0} \cap \Omega_{n}, \quad n \geqslant n_{0} .
$$

Suppose, in addition, that $a=\mathcal{X}$ and $\lambda \in \Lambda\left[\gamma, \Omega_{0}, \mathfrak{B}_{0}(b)\right]$. Then, $m_{0} \in \mathbb{N}, m_{0} \geqslant$ $n_{0}$, exists for which

$$
\lambda \in \bigcap_{n=m_{0}}^{\infty} \Lambda\left[\gamma, \Omega_{n}, \mathfrak{B}_{n}(b)\right] .
$$

(d) Suppose (2.8) on $\Gamma_{1} \cap \partial\left[\Omega_{0}\right]_{a}^{0}$ and

(1) $\Omega_{a}^{0} \cap \Omega_{0} \neq \emptyset$ is of class $\mathcal{C}^{2}$,

(2) $\Omega_{a}^{0} \cap\left(\Omega \backslash \Omega_{0}\right) \neq \emptyset$,

(3) $n_{0} \in \mathbb{N}$ exists such that $\Omega_{a}^{0} \cap \Omega_{n}$ is a proper subdomain of $\Omega$ of class $\mathcal{C}^{2}$ if $n \geqslant n_{0}$

(4) (2.14) is satisfied for any $\widetilde{\Omega} \in\left\{\Omega_{0}, \Omega_{n_{0}+j}: j \geqslant 0\right\}$.

Then, $m_{0} \geqslant n_{0}$ exists for which

$$
a \in \bigcap_{n=m_{0}}^{\infty} \mathcal{A}_{\Gamma_{0}^{n}, \Gamma_{1}}\left(\Omega_{n}\right) \wedge\left[\Omega_{n}\right]_{a}^{0}=\Omega_{n} \cap \Omega_{a}^{0} \text { if } n \in\left\{0, m_{0}+j: j \geqslant 0\right\} .
$$

Moreover, if, in addition, $a=\mathcal{X}$ and $\lambda \in \Lambda\left[\gamma, \Omega_{0}, \mathfrak{B}_{0}(b)\right]$, then, for some $\ell_{0} \geqslant$ $m_{0}$,

$$
\lambda \in \bigcap_{n=\ell_{0}}^{\infty} \Lambda\left[\gamma, \Omega_{n}, \mathfrak{B}_{n}(b)\right] .
$$

(e) Suppose $a \in \mathcal{A}_{\Gamma_{0}, \Gamma_{1}}^{+}(\Omega)$, i.e., $\Omega_{a}^{0}=\emptyset$. Then,

$$
a \in \bigcap_{n=0}^{\infty} \mathcal{A}_{\Gamma_{0}^{n}, \Gamma_{1}}^{+}\left(\Omega_{n}\right)
$$

i.e., $a \in \mathcal{A}_{\Gamma_{0}^{n}, \Gamma_{1}}\left(\Omega_{n}\right)$ and $\left[\Omega_{n}\right]_{a}^{0}=\emptyset$ for each $n \geqslant 0$. Moreover,

$$
a=\mathcal{X} \wedge \lambda \in \Lambda\left[\gamma, \Omega_{0}, \mathfrak{B}_{0}(b)\right] \Longrightarrow \lambda \in \bigcap_{n=0}^{\infty} \Lambda\left[\gamma, \Omega_{n}, \mathfrak{B}_{n}(b)\right]
$$

Furthermore, in any of the five previous cases, if $a=\mathcal{X}$, then

$$
\lim _{n \rightarrow \infty}\left\|\left.u_{\left[\mathfrak{L}+\gamma V, \lambda W, \mathcal{X}, \Omega_{n}, \mathfrak{B}_{n}(b)\right]}\right|_{\Omega_{0}}-u_{\left[\mathfrak{L}+\gamma V, \lambda W, \mathcal{X}, \Omega_{0}, \mathfrak{B}_{0}(b)\right]}\right\|_{H^{1}\left(\Omega_{0}\right)}=0
$$

if

$$
\lambda \in \Lambda\left[\gamma, \Omega_{0}, \mathfrak{B}_{0}(b)\right]
$$


S. CANO-CASANOVA, J. LÓPEZ-GÓMEZ / Ann. I. H. Poincaré - AN 20 (2003) 999-1041 1015

\section{Proof of Theorem 1.3}

This section proves Theorem 1.3. Subsequently, for any

$$
a \in \mathcal{A}_{\partial \Omega_{V}^{0} \backslash \Gamma_{1}, \partial \Omega_{V}^{0} \cap \Gamma_{1}}\left(\Omega_{V}^{0}\right) \cap \mathcal{A}_{\Gamma_{0}, \Gamma_{1}}(\Omega)
$$

satisfying $\Omega_{a}^{0} \cap \Omega_{V}^{0} \neq \emptyset$ we set

$$
\Omega_{a, V}^{0}:=\left[\Omega_{V}^{0}\right]_{a}^{0}=\left[\Omega_{a}^{0}\right]_{V}^{0}=\Omega_{a}^{0} \cap \Omega_{V}^{0} .
$$

Our proof of Theorem 1.3 is based upon the following proposition.

Proposition 3.1. - Suppose

$$
\lambda \in \Lambda\left[0, \Omega_{V}^{0}, \mathfrak{B}\left(b, \Omega_{V}^{0}\right)\right]
$$

and (2.8) holds on $\Gamma_{1} \cap \partial \Omega_{V}^{0}$.

Then, $\delta_{0}>0$ exists such that for each $\delta \in\left(0, \delta_{0}\right)$ there are a real number $\Lambda(\delta)>0$ and a positive function $\bar{u}_{\delta}$ satisfying the following conditions:

(i) $\bar{u}_{\delta}$ is a positive strict supersolution of $P[\gamma, \lambda, \Omega, \mathfrak{B}(b)]$ for each $\gamma>\Lambda(\delta)$.

(ii) One has that

$$
\lim _{\delta \searrow 0}\left\|\bar{u}_{\delta}-u_{\left[\mathfrak{L}, \lambda W, \mathcal{X}, \Omega_{V}^{0}, \mathfrak{B}\left(b, \Omega_{V}^{0}\right)\right]}\right\|_{H^{1}\left(\Omega_{V}^{0}\right)}=0
$$

and, for any compact subset $K \subset \bar{\Omega} \backslash \bar{\Omega}_{V}^{0}$,

$$
\lim _{\delta \searrow 0}\left\|\bar{u}_{\delta}\right\|_{L_{\infty}(K)}=0 .
$$

In particular,

$$
\lim _{\delta \searrow 0} \bar{u}_{\delta}=\left\{\begin{array}{ll}
u_{\left[\mathfrak{L}, \lambda W, \mathcal{X}, \Omega_{V}^{0}, \mathfrak{B}\left(b, \Omega_{V}^{0}\right)\right]} & \text { in } \Omega_{V}^{0}, \\
0 & \text { in } \Omega \backslash \Omega_{V}^{0}
\end{array} \quad \text { a.e. in } \Omega .\right.
$$

Proof. - Firstly, we shall prove part (i) in case

$$
\Gamma_{0} \cap K_{V}=\emptyset .
$$

Then, since $V \in \mathcal{A}_{\Gamma_{0}, \Gamma_{1}}(\Omega)$, we have that

$$
K_{V} \cap\left(\bar{\Omega}_{V}^{0} \cup \Gamma_{1}\right)=\emptyset
$$

and, hence, (3.5) gives

$$
K_{V} \cap \partial \Omega=\emptyset .
$$

Thus, taking into account (1.7), we have that

$$
K_{V} \subset \Omega, \quad K_{V} \cap \bar{\Omega}_{V}^{0}=\emptyset, \quad \bar{\Omega}_{V}^{0} \subset \Omega \cup \Gamma_{1},
$$


1016 S. CANO-CASANOVA, J. LÓPEZ-GÓMEZ / Ann. I. H. Poincaré - AN 20 (2003) 999-1041

and, in particular,

$$
\operatorname{dist}\left(\Gamma_{0}, \bar{\Omega}_{V}^{0} \cup K_{V}\right)>0, \quad \operatorname{dist}\left(\Gamma_{1}, K_{V}\right)>0, \quad \operatorname{dist}\left(K_{V}, \bar{\Omega}_{V}^{0}\right)>0 .
$$

Fix $\eta>0$. Since $V \in \mathcal{A}_{\Gamma_{0}, \Gamma_{1}}(\Omega)$, it follows from $\left(\mathcal{A}_{4}\right)$, that there exist a natural number $\ell_{V}(\eta) \geqslant 1$ and $\ell_{V}(\eta)$ open sets

$$
G_{j}^{\eta} \subset \mathbb{R}^{N}, \quad 1 \leqslant j \leqslant \ell_{V}(\eta),
$$

such that

$$
\begin{aligned}
& \left|G_{j}^{\eta}\right|<\eta, \quad 1 \leqslant j \leqslant \ell_{V}(\eta), \\
& K_{V} \subset \bigcup_{j=1}^{\ell_{V}(\eta)} G_{j}^{\eta} \wedge \quad \bar{G}_{i}^{\eta} \cap \bar{G}_{j}^{\eta}=\emptyset \text { if } i \neq j
\end{aligned}
$$

and for each $1 \leqslant j \leqslant \ell_{V}(\eta)$ the open set $G_{j}^{\eta} \cap \Omega$ is connected and of class $\mathcal{C}^{2}$. Thanks to (3.6), the $G_{j}^{\eta}$,s can be chosen so that

$$
K_{V} \subset \bigcup_{j=1}^{\ell_{V}(\eta)} \bar{G}_{j}^{\eta} \subset \Omega, \quad \bigcup_{j=1}^{\ell_{V}(\eta)} \bar{G}_{j}^{\eta} \cap \bar{\Omega}_{V}^{0}=\emptyset .
$$

Indeed, since

$$
\operatorname{dist}\left(K_{V}, \bar{\Omega}_{V}^{0} \cup \Gamma_{0} \cup \Gamma_{1}\right)>0,
$$

an open set $G$ exists such that

$$
K_{V} \subset G, \quad \bar{G} \subset \Omega, \quad \bar{G} \cap \bar{\Omega}_{V}^{0}=\emptyset,
$$

and, hence, in order to have (3.8), it suffices considering $G \cap G_{j}^{\eta}$ instead of $G_{j}^{\eta}, 1 \leqslant j \leqslant$ $\ell_{V}(\eta)$

Thanks to (3.8), there exist $\varepsilon:=\varepsilon(\eta)>0$ and $\ell_{V}(\eta)$ open sets $G_{j}^{\eta, \varepsilon}, 1 \leqslant j \leqslant \ell_{V}(\eta)$, of class $\mathcal{C}^{2}$ such that

$$
\bar{G}_{j}^{\eta} \subset G_{j}^{\eta, \varepsilon} \subset G_{j}^{\eta}+B_{\varepsilon}, \quad\left|G_{j}^{\eta, \varepsilon}\right|<2 \eta, \quad 1 \leqslant j \leqslant \ell_{V}(\eta),
$$

and

$$
K_{V} \subset \bigcup_{j=1}^{\ell_{V}(\eta)} \bar{G}_{j}^{\eta} \subset \bigcup_{j=1}^{\ell_{V}(\eta)} G_{j}^{\eta, \varepsilon} \subset \Omega, \quad \bigcup_{j=1}^{\ell_{V}(\eta)} \bar{G}_{j}^{\eta, \varepsilon} \cap \bar{\Omega}_{V}^{0}=\emptyset,
$$

where, for any $\varrho>0, B_{\varrho}$ stands for the ball of radius $\varrho$ centered at zero. Since

$$
\lim _{\eta \backslash 0}\left|G_{j}^{\eta, \varepsilon}\right|=0, \quad 1 \leqslant j \leqslant \ell_{V}(\eta),
$$

it follows from Theorem 2.8 that $\eta_{0}>0$ exists such that for each $\eta \in\left(0, \eta_{0}\right)$ and $1 \leqslant j \leqslant \ell_{V}(\eta)$ we have that

$$
\min _{1 \leqslant j \leqslant \ell_{V}(\eta)} \sigma\left[\mathfrak{L}+\mathcal{X} f(\cdot, 0)-\lambda W, G_{j}^{\eta, \varepsilon(\eta)}, \mathfrak{D}\right]>0 .
$$


S. CANO-CASANOVA, J. LÓPEZ-GÓMEZ / Ann. I. H. Poincaré - AN 20 (2003) 999-1041 1017

Subsequently we consider $\eta \in\left(0, \eta_{0}\right)$ fixed. For each $k \in\{0,1\}$, let $\Gamma_{k}^{j}, 1 \leqslant j \leqslant n_{k}$, denote the components of $\Gamma_{k}$. Let $\left\{i_{1}, \ldots, i_{p}\right\}$ denote the subset of $\left\{1, \ldots, n_{1}\right\}$ for which

$$
\Gamma_{1}^{j} \cap \partial \Omega_{V}^{0}=\emptyset \quad \Longleftrightarrow j \in\left\{i_{1}, \ldots, i_{p}\right\} .
$$

Since $V \in \mathcal{A}_{\Gamma_{0}, \Gamma_{1}}(\Omega)$, we find from $\left(\mathcal{A}_{1}\right)$, that $\Gamma_{1}^{j}$ is a component of $\partial \Omega_{V}^{0}$ for each $j \in\left\{1, \ldots, n_{1}\right\} \backslash\left\{i_{1}, \ldots, i_{p}\right\}$. Then,

$$
\Gamma_{1} \cap \partial \Omega_{V}^{0}=\bigcup_{j \in\left\{1, \ldots, n_{1}\right\}\left\{i_{1}, \ldots, i_{p}\right\}} \Gamma_{1}^{j} \wedge \bigcup_{j=1}^{p} \Gamma_{1}^{i_{j}} \cap \partial \Omega_{V}^{0}=\emptyset .
$$

In particular,

$$
\operatorname{dist}\left(\bigcup_{j=1}^{p} \Gamma_{1}^{i_{j}}, \partial \Omega_{V}^{0}\right)>0
$$

Subsequently, for each

$$
\delta \in\left(0, \operatorname{dist}\left(\Gamma_{0}^{0}, \partial \Omega\right)\right)
$$

we consider the open $\delta$-neighborhood

$$
\Omega_{V}^{\delta}:=\Omega_{V}^{0} \cup\left\{x \in \Omega: \operatorname{dist}\left(x, \Gamma_{0}^{0}\right)<\delta\right\} .
$$

By definition, for any sequence $\delta_{n}, n \geqslant 1$, such that

$$
\lim _{n \rightarrow \infty} \delta_{n}=0
$$

we have that the sequence

$$
\Omega_{n}:=\Omega_{V}^{\delta_{n}}, \quad n \geqslant 1,
$$

converges to $\Omega_{V}^{0}$ from the exterior as $n \rightarrow \infty$; it will be simply said that

$$
\lim _{\delta \searrow 0} \Omega_{V}^{\delta}=\Omega_{V}^{0} \quad \text { from the exterior. }
$$

By construction, for each $\delta>0$ sufficiently small, we have

$$
\Gamma_{0}^{0} \cup \Omega_{V}^{0} \subset \Omega_{V}^{\delta} \subset \Omega
$$

and

$$
\partial \Omega_{V}^{0} \cap \Gamma_{1}=\partial \Omega_{V}^{\delta} \cap \Gamma_{1}=\bigcup_{j \in\left\{1, \ldots, n_{1}\right\} \backslash\left\{i_{1}, \ldots, i_{p}\right\}} \Gamma_{1}^{j} .
$$

Now, for each $\delta>0$ sufficiently small, we consider the $\delta$-neighborhoods

$$
\begin{array}{ll}
\mathcal{N}_{\delta}^{0, j}:=\left(\Gamma_{0}^{j}+B_{\delta}\right) \cap \Omega, & 1 \leqslant j \leqslant n_{0}, \\
\mathcal{N}_{\delta}^{1, j}:=\left(\Gamma_{1}^{j}+B_{\delta}\right) \cap \Omega, & j \in\left\{i_{1}, \ldots, i_{p}\right\} .
\end{array}
$$


Since

$$
K_{V} \cap\left(\bar{\Omega}_{V}^{0} \cup \Gamma_{1}\right)=\emptyset, \quad \Omega_{V}^{+}=\Omega \backslash\left(\bar{\Omega}_{V}^{0} \cup K_{V}\right),
$$

it follows from (1.7), (3.5), (3.10) and (3.12) that $\delta_{1}>0$ exists such that, for any $\delta \in\left(0, \delta_{1}\right)$,

$$
\bigcup_{j=1}^{\ell_{V}(\eta)} \bar{G}_{j}^{\eta, \varepsilon} \cap \bar{\Omega}_{V}^{\delta}=\emptyset, \quad \partial \Omega_{V}^{\delta} \backslash \Gamma_{1} \subset \Omega_{V}^{+}, \quad \bigcup_{j=1}^{n_{0}} \overline{\mathcal{N}}_{\delta}^{0, j} \backslash \Gamma_{0} \subset \Omega_{V}^{+}
$$

and

$$
\left(\bigcup_{j=1}^{p} \overline{\mathcal{N}}_{\delta}^{1, i_{j}} \cup \bigcup_{j=1}^{n_{0}} \overline{\mathcal{N}}_{\delta}^{0, j}\right) \cap\left(\bar{\Omega}_{V}^{\delta} \cup \bigcup_{j=1}^{\ell_{V}(\eta)} \bar{G}_{j}^{\eta, \varepsilon}\right)=\emptyset .
$$

Moreover, since $\Gamma_{k}^{j} \cap \Gamma_{\ell}^{i}=\emptyset$ if $(i, \ell) \neq(j, k), \delta_{2} \in\left(0, \delta_{1}\right)$ exists such that for each $0<\delta<\delta_{2}$

$$
\overline{\mathcal{N}}_{\delta}^{k, j} \cap \overline{\mathcal{N}}_{\delta}^{\ell, i}=\emptyset \quad \text { if }(i, \ell) \neq(j, k), k, \ell \in\{0,1\} .
$$

Furthermore, since

$$
\lim _{\delta \searrow 0}\left|\mathcal{N}_{\delta}^{0, j}\right|=0, \quad 1 \leqslant j \leqslant n_{0},
$$

it follows from Theorem 2.8 that $\delta_{3} \in\left(0, \delta_{2}\right)$ exists such that for each $0<\delta<\delta_{3}$

$$
\sigma\left[\mathfrak{L}+\mathcal{X} f(\cdot, 0)-\lambda W, \mathcal{N}_{\delta}^{0, j}, \mathfrak{D}\right]>0, \quad 1 \leqslant j \leqslant n_{0} .
$$

Since

$$
\lambda \in \Lambda\left[0, \Omega_{V}^{0}, \mathfrak{B}\left(b, \Omega_{V}^{0}\right)\right],
$$

taking into account the general assumptions in Section 1, it follows from Theorem 2.19 that there exists $\delta_{4} \in\left(0, \delta_{3}\right)$ such that

$$
\lambda \in \Lambda\left[0, \Omega_{V}^{\delta_{4}}, \mathfrak{B}\left(b, \Omega_{V}^{\delta_{4}}\right)\right] .
$$

For each $\delta \geqslant 0$ sufficiently small let

$$
u_{\delta}:=u_{\left[\mathfrak{L}, \lambda W, \mathcal{X}, \Omega_{V}^{\delta}, \mathfrak{B}\left(b, \Omega_{V}^{\delta}\right)\right]}
$$

denote the unique positive solution of $P\left[0, \lambda, \Omega_{V}^{\delta}, \mathfrak{B}\left(b, \Omega_{V}^{\delta}\right)\right]$, if it exists; the uniqueness is a consequence from Theorem 2.12, since we are assuming that (2.8) holds on $\Gamma_{1} \cap \partial \Omega_{V}^{0}$ and, by construction, we have (1.8) and

$$
\Gamma_{1} \cap \partial\left[\Omega_{V}^{\delta}\right]_{\mathcal{X}}^{0} \subset \Gamma_{1} \cap \partial \Omega_{V}^{0} .
$$

It is easy to see that $u_{\delta_{4}}$ is a positive supersolution of $P\left[0, \lambda, \Omega_{V}^{\delta}, \mathfrak{B}\left(b, \Omega_{V}^{\delta}\right)\right]$ if $\delta \in$ $\left(0, \delta_{4}\right)$. In fact, thanks to the monotone structure of the nonlinearity, for any $\kappa \geqslant 1, \kappa u_{\delta_{4}}$ 
S. CANO-CASANOVA, J. LÓPEZ-GÓMEZ / Ann. I. H. Poincaré - AN 20 (2003) 999-1041 1019

is a positive supersolution of $P\left[0, \lambda, \Omega_{V}^{\delta}, \mathfrak{B}\left(b, \Omega_{V}^{\delta}\right)\right]$. On the other hand, it is easy to see that, for any $\delta \in\left(0, \delta_{4}\right)$,

$$
v_{\delta}:= \begin{cases}u_{0} & \text { in } \Omega_{V}^{0}, \\ 0 & \text { in } \Omega_{V}^{\delta} \backslash \Omega_{V}^{0}\end{cases}
$$

is a subsolution of $P\left[0, \lambda, \Omega_{V}^{\delta}, \mathfrak{B}\left(b, \Omega_{V}^{\delta}\right)\right]$ such that

$$
v_{\delta} \leqslant \kappa u_{\delta}
$$

for each $\kappa>1$ sufficiently large. Therefore,

$$
\lambda \in \bigcap_{0<\delta<\delta_{4}} \Lambda\left[0, \Omega_{V}^{\delta}, \mathfrak{B}\left(b, \Omega_{V}^{\delta}\right)\right]
$$

In other words, $u_{\delta}$ exists - and it is unique - for any $\delta \in\left(0, \delta_{4}\right)$.

Subsequently, we fix $\delta \in\left(0, \delta_{4}\right)$ and set

$$
H_{\delta / 2}^{\eta}:=\bigcup_{j=1}^{\ell_{V}(\eta)} \bar{G}_{j}^{\eta} \cup \bigcup_{j=1}^{p} \overline{\mathcal{N}}_{\delta / 2}^{1, i_{j}} \cup \bigcup_{j=1}^{n_{0}} \overline{\mathcal{N}}_{\delta / 2}^{0, j}
$$

Let $\psi_{\delta}^{i}, i \in\left\{i_{1}, \ldots, i_{p}\right\}$, and $\xi_{\delta}^{j}, 1 \leqslant j \leqslant n_{0}$, denote the principal eigenfunctions associated with

$$
\sigma\left[\mathfrak{L}+\mathcal{X} f(\cdot, 0)-\lambda W, \mathcal{N}_{\delta}^{1, i}, \mathfrak{B}\left(b, \mathcal{N}_{\delta}^{1, i}\right)\right], \quad i \in\left\{i_{1}, \ldots, i_{p}\right\},
$$

and

$$
\sigma\left[\mathfrak{L}+\mathcal{X} f(\cdot, 0)-\lambda W, \mathcal{N}_{\delta}^{0, j}, \mathfrak{D}\right], \quad 1 \leqslant j \leqslant n_{0},
$$

respectively, normalized so that

$$
\left\|\psi_{\delta}^{i}\right\|_{L_{\infty}\left(\mathcal{N}_{\delta}^{1, i}\right)}=1, \quad\left\|\xi_{\delta}^{j}\right\|_{L_{\infty}\left(\mathcal{N}_{\delta}^{0, j}\right)}=1, \quad i \in\left\{i_{1}, \ldots, i_{p}\right\}, 1 \leqslant j \leqslant n_{0},
$$

and let $\vartheta_{\delta}^{j}, 1 \leqslant j \leqslant \ell_{V}(\eta)$, denote the principal eigenfunctions associated with

$$
\sigma\left[\mathfrak{L}+\mathcal{X} f(\cdot, 0)-\lambda W, G_{j}^{\eta, \varepsilon}, \mathfrak{D}\right]
$$

normalized so that

$$
\left\|\vartheta_{\delta}^{j}\right\|_{L_{\infty}\left(G_{j}^{\eta, \varepsilon}\right)}=1, \quad 1 \leqslant j \leqslant \ell_{V}(\eta) .
$$

Now, consider the positive function

$$
\bar{u}_{\delta}: \bar{\Omega} \rightarrow[0, \infty)
$$


defined by

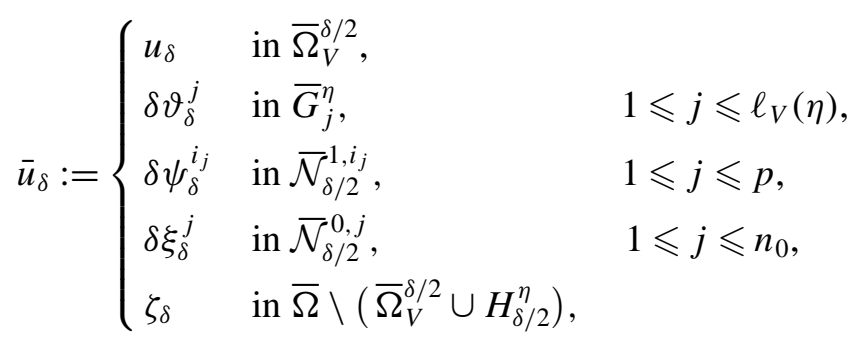

where $\zeta_{\delta}$ is any positive regular extension of the function

$$
u_{\delta} \cup \bigcup_{j=1}^{\ell_{V}(\eta)} \delta \vartheta_{\delta}^{j} \cup \bigcup_{j=1}^{p} \delta \psi_{\delta}^{i_{j}} \cup \bigcup_{j=1}^{n_{0}} \delta \xi_{\delta}^{j}
$$

from $\bar{\Omega}_{V}^{\delta / 2} \cup H_{\delta / 2}^{\eta}$ to $\bar{\Omega}$ with the property of being bounded away from zero in $\bar{\Omega} \backslash\left(\bar{\Omega}_{V}^{\delta / 2} \cup\right.$ $\left.H_{\delta / 2}^{\eta}\right) ; \zeta_{\delta}$ exists since each of the functions

$$
\begin{array}{ll}
\left.u_{\delta}\right|_{\partial \Omega_{V}^{\delta / 2} \backslash \Gamma_{1}}, & \left.\vartheta_{\delta}^{j}\right|_{\partial G_{j}^{\eta}}, \quad 1 \leqslant j \leqslant \ell_{V}(\eta), \\
\left.\psi_{\delta}^{i_{j}}\right|_{\partial \mathcal{N}_{\delta / 2}^{1, i_{j}} \backslash \Gamma_{1}},\left.\quad \xi_{\delta}^{i}\right|_{\partial \mathcal{N}_{\delta / 2}^{0, i} \backslash \Gamma_{0}}, \quad 1 \leqslant j \leqslant p, 1 \leqslant i \leqslant n_{0},
\end{array}
$$

is positive and bounded away from zero. When $\Gamma_{1} \subset \partial \Omega_{V}^{0}$, one should remove the $\psi_{\delta}^{i_{j}}$,s, $1 \leqslant j \leqslant p$, from the definition of $\bar{u}_{\delta}$. It should be noted that, thanks to (3.17), (3.18) and (3.19), the function $\bar{u}_{\delta}$ is well defined. Moreover,

$$
\bar{u}_{\delta}(x)>0 \quad \text { for each } x \in \Omega \text {. }
$$

To complete the proof of part (i) when (3.5) occurs it remains to show that $\Lambda=\Lambda(\delta)>0$ exists such that $\bar{u}_{\delta}$ provides us with a strict supersolution of $P[\gamma, \lambda, \Omega, \mathfrak{B}(b)]$ for each $\gamma>\Lambda(\delta)$. Indeed, since

$$
V \geqslant 0 \wedge \Omega_{V}^{0} \subset \Omega_{V}^{\delta / 2}
$$

we find that, in $\Omega_{V}^{\delta / 2}$, the following estimate is satisfied for any $\gamma>0$

$$
\left[\mathfrak{L}+\gamma V-\lambda W+\mathcal{X} f\left(\cdot, \bar{u}_{\delta}\right)\right] \bar{u}_{\delta}=\left[\mathfrak{L}+\gamma V-\lambda W+\mathcal{X} f\left(\cdot, u_{\delta}\right)\right] u_{\delta}=\gamma V u_{\delta}>0,
$$

by construction. Also, since $\delta \vartheta_{\delta}^{j}>0$ in $G_{j}^{\eta}$ for each $1 \leqslant j \leqslant \ell_{V}(\eta)$, it follows from (1.2) that

$$
f\left(\cdot, \delta \vartheta_{\delta}^{j}\right)>f(\cdot, 0) \quad \text { in } G_{j}^{\eta},
$$

and, hence, for each $1 \leqslant j \leqslant \ell_{V}(\eta)$ and $\gamma>0$ the following estimate holds in $G_{j}^{\eta}$

$$
\begin{aligned}
& {\left[\mathfrak{L}+\gamma V-\lambda W+\mathcal{X} f\left(\cdot, \bar{u}_{\delta}\right)\right] \bar{u}_{\delta}=\delta\left[\mathfrak{L}+\gamma V-\lambda W+\mathcal{X} f\left(\cdot, \delta \vartheta_{\delta}^{j}\right)\right] \vartheta_{\delta}^{j}} \\
& \quad=\delta\left\{\sigma\left[\mathfrak{L}+\mathcal{X} f(\cdot, 0)-\lambda W, G_{j}^{\eta, \varepsilon}, \mathfrak{D}\right]+\gamma V+\mathcal{X}\left[f\left(\cdot, \delta \vartheta_{\delta}^{j}\right)-f(\cdot, 0)\right]\right\} \vartheta_{\delta}^{j} \\
& \quad \geqslant \delta \sigma\left[\mathfrak{L}+\mathcal{X} f(\cdot, 0)-\lambda W, G_{j}^{\eta, \varepsilon}, \mathfrak{D}\right] \vartheta_{\delta}^{j} .
\end{aligned}
$$


S. CANO-CASANOVA, J. LÓPEZ-GÓMEZ / Ann. I. H. Poincaré - AN 20 (2003) 999-1041 1021

Thus, it follows from (3.11) that, for any $\gamma>0$,

$$
\left[\mathfrak{L}+\gamma V-\lambda W+\mathcal{X} f\left(\cdot, \bar{u}_{\delta}\right)\right] \bar{u}_{\delta}>0 \quad \text { in } G_{j}^{\eta}, 1 \leqslant j \leqslant \ell_{V}(\eta) .
$$

Similarly, since $\delta \xi_{\delta}^{j}>0$ in $\mathcal{N}_{\delta / 2}^{0, j}$ for each $1 \leqslant j \leqslant n_{0},(1.2)$ implies

$$
f\left(\cdot, \delta \xi_{\delta}^{j}\right)>f(\cdot, 0) \quad \text { in } \mathcal{N}_{\delta / 2}^{0, j}, 1 \leqslant j \leqslant n_{0},
$$

and, hence, for each $1 \leqslant j \leqslant n_{0}$ and $\gamma>0$ the following estimate is satisfied in $\mathcal{N}_{\delta / 2}^{0, j}$,

$$
\begin{aligned}
& {\left[\mathfrak{L}+\gamma V-\lambda W+\mathcal{X} f\left(\cdot, \bar{u}_{\delta}\right)\right] \bar{u}_{\delta}=\delta\left[\mathfrak{L}+\gamma V-\lambda W+\mathcal{X} f\left(\cdot, \delta \xi_{\delta}^{j}\right)\right] \xi_{\delta}^{j}} \\
& \quad=\delta\left\{\sigma\left[\mathfrak{L}-\lambda W+\mathcal{X} f(\cdot, 0), \mathcal{N}_{\delta}^{0, j}, \mathfrak{D}\right]+\gamma V+\mathcal{X}\left[f\left(\cdot, \delta \xi_{\delta}^{j}\right)-f(\cdot, 0)\right]\right\} \xi_{\delta}^{j} \\
& \quad \geqslant \delta \sigma\left[\mathfrak{L}-\lambda W+\mathcal{X} f(\cdot, 0), \mathcal{N}_{\delta}^{0, j}, \mathfrak{D}\right] \xi_{\delta}^{j} .
\end{aligned}
$$

Thus, thanks to (3.20), for each $\gamma>0$ the following estimate is satisfied

$$
\left[\mathfrak{L}+\gamma V-\lambda W+\mathcal{X} f\left(\cdot, \bar{u}_{\delta}\right)\right] \bar{u}_{\delta}>0 \quad \text { in } \mathcal{N}_{\delta / 2}^{0, j}, 1 \leqslant j \leqslant n_{0} .
$$

Summarizing, up to now we have shown that, for each $\delta \in\left(0, \delta_{4}\right)$ and $\gamma>0$,

$$
\left[\mathfrak{L}+\gamma V-\lambda W+\mathcal{X} f\left(\cdot, \bar{u}_{\delta}\right)\right] \bar{u}_{\delta}>0 \quad \text { in } \bar{\Omega}_{V}^{\delta / 2} \cup \bigcup_{j=1}^{\ell_{V}(\eta)} \bar{G}_{j}^{\eta} \cup \bigcup_{j=1}^{n_{0}} \overline{\mathcal{N}}_{\delta / 2}^{0, j}
$$

Now, since $V \in \mathcal{A}_{\Gamma_{0}, \Gamma_{1}}(\Omega)$, due to $\left(\mathcal{A}_{2}\right)$ a constant $\omega>0$ exists such that

$$
V>\omega>0 \quad \text { in any compact subset of } \Omega_{V}^{+} \cup \bigcup_{j=1}^{p} \Gamma_{1}^{i_{j}}
$$

and, hence,

$$
V>\omega>0 \quad \text { in }\left[\bar{\Omega} \backslash\left(\bar{\Omega}_{V}^{\delta / 2} \cup H_{\delta / 2}^{\eta}\right)\right] \cup \bigcup_{j=1}^{p} \mathcal{N}_{\delta / 2}^{1, i_{j}} \subset \Omega_{V}^{+} \cup \bigcup_{j=1}^{p} \Gamma_{1}^{i_{j}} .
$$

Thus, since $\delta \psi_{\delta}^{i_{j}}>0$ in $\mathcal{N}_{\delta / 2}^{1, i_{j}}$ for each $1 \leqslant j \leqslant p$, we find from (1.2) that

$$
f\left(\cdot, \delta \psi_{\delta}^{i_{j}}\right)>f(\cdot, 0) \quad \text { in } \mathcal{N}_{\delta / 2}^{1, i_{j}}, 1 \leqslant j \leqslant p
$$

Hence, thanks to (3.28), the following estimate is satisfied in $\mathcal{N}_{\delta / 2}^{1, i_{j}}$ for each $1 \leqslant j \leqslant p$

$$
\begin{aligned}
{[\mathfrak{L}+} & \left.\gamma V-\lambda W+\mathcal{X} f\left(\cdot, \bar{u}_{\delta}\right)\right] \bar{u}_{\delta}=\delta\left[\mathfrak{L}+\gamma V-\lambda W+\mathcal{X} f\left(\cdot, \delta \psi_{\delta}^{i_{j}}\right)\right] \psi_{\delta}^{i_{j}} \\
=\delta\left\{\sigma\left[\mathfrak{L}+\mathcal{X} f(\cdot, 0)-\lambda W, \mathcal{N}_{\delta}^{1, i_{j}}, \mathfrak{B}\left(b, \mathcal{N}_{\delta}^{1, i_{j}}\right)\right]\right. & \left.+\gamma V+\mathcal{X}\left[f\left(\cdot, \delta \psi_{\delta}^{i_{j}}\right)-f(\cdot, 0)\right]\right\} \psi_{\delta}^{i_{j}} \\
& \left.+\gamma\left(\mathcal{N}^{1, i_{j}}, \mathfrak{B}\left(b, \mathcal{N}_{\delta}^{1, i_{j}}\right)\right]+\gamma \omega\right\} \psi_{\delta}^{i_{j}}
\end{aligned}
$$


and, therefore,

$$
\left[\mathfrak{L}+\gamma V-\lambda W+\mathcal{X} f\left(\cdot, \bar{u}_{\delta}\right)\right] \bar{u}_{\delta}>0 \quad \text { in } \bigcup_{j=1}^{p} \mathcal{N}_{\delta / 2}^{1, i_{j}}
$$

if

$$
\gamma>\Lambda_{1}(\delta):=\omega^{-1} \max _{1 \leqslant j \leqslant p}\left\{\left|\sigma\left[\mathfrak{L}+\mathcal{X} f(\cdot, 0)-\lambda W, \mathcal{N}_{\delta}^{1, i_{j}}, \mathfrak{B}\left(b, \mathcal{N}_{\delta}^{1, i_{j}}\right)\right]\right|\right\} \geqslant 0 .
$$

Moreover, since

$$
\bar{\Omega} \backslash\left(\bar{\Omega}_{V}^{\delta / 2} \cup H_{\delta / 2}^{\eta}\right) \subset \Omega_{V}^{+},
$$

it follows from (3.28) that there exists

$$
\Lambda(\delta)>\max \left\{\Lambda_{1}(\delta), 0\right\}
$$

such that for each $\gamma>\Lambda(\delta)$ the following estimates are satisfied in $\bar{\Omega} \backslash\left(\bar{\Omega}_{V}^{\delta / 2} \cup H_{\delta / 2}^{\eta}\right)$

$$
\begin{aligned}
{\left[\mathfrak{L}+\gamma V-\lambda W+\mathcal{X} f\left(\cdot, \bar{u}_{\delta}\right)\right] \bar{u}_{\delta} } & =\left[\mathfrak{L}+\gamma V-\lambda W+\mathcal{X} f\left(\cdot, \zeta_{\delta}\right)\right] \zeta_{\delta} \\
& >\left[\mathfrak{L}-\lambda W+\mathcal{X} f\left(\cdot, \zeta_{\delta}\right)+\gamma \omega\right] \zeta_{\delta}>0,
\end{aligned}
$$

because $\omega>0, \zeta_{\delta}$ is bounded away from zero in $\bar{\Omega} \backslash\left(\bar{\Omega}_{V}^{\delta / 2} \cup H_{\delta / 2}^{\eta}\right)$ and the function

$$
\left[\mathfrak{L}-\lambda W+\mathcal{X} f\left(\cdot, \zeta_{\delta}\right)\right] \zeta_{\delta}
$$

is independent of $\gamma$.

On the other hand, by construction, we have that

$$
\begin{aligned}
& \mathfrak{B}(b) \bar{u}_{\delta}=\delta \mathfrak{D} \xi_{\delta}^{j}=0 \quad \text { on } \Gamma_{0}^{j}, 1 \leqslant j \leqslant n_{0}, \\
& \mathfrak{B}(b) \bar{u}_{\delta}=\delta\left(\partial_{\nu}+b\right) \psi_{\delta}^{i_{j}}=0 \quad \text { on } \Gamma_{1}^{i_{j}}, 1 \leqslant j \leqslant p,
\end{aligned}
$$

and, thanks to (3.15),

$$
\mathfrak{B}(b) \bar{u}_{\delta}=\left(\partial_{v}+b\right) u_{\delta}=0 \quad \text { on } \partial \Omega_{V}^{0} \cap \Gamma_{1} .
$$

Therefore,

$$
\mathfrak{B}(b) \bar{u}_{\delta}=0 \quad \text { on } \partial \Omega
$$

and, for each $\delta \in\left(0, \delta_{4}\right)$ and $\gamma>\Lambda(\delta)$, the function $\bar{u}_{\delta}$ provides us with a positive strict supersolution of $P[\gamma, \lambda, \Omega, \mathfrak{B}(b)]$. This completes the proof of part (i) under condition (3.5).

Now, suppose

$$
\Gamma_{0} \cap K_{V} \neq \varnothing,
$$

instead of (3.5), and let $\left\{i_{1}, \ldots, i_{q}\right\}$ be the subset of $\left\{1, \ldots, n_{0}\right\}$ for which

$$
\Gamma_{0}^{j} \cap K_{V} \neq \emptyset \Longleftrightarrow j \in\left\{i_{1}, \ldots, i_{q}\right\} .
$$


S. CANO-CASANOVA, J. LÓPEZ-GÓMEZ / Ann. I. H. Poincaré - AN 20 (2003) 999-1041 1023

Subsequently, for any $\varrho>0$ sufficiently small we will consider the new support domain

$$
\mathfrak{O}_{\varrho}:=\Omega \cup\left(\bigcup_{j=1}^{q} \Gamma_{0}^{i_{j}}+B_{\varrho}\right) .
$$

Fix $\varrho_{1}>0$, let

$$
\tilde{\alpha}_{i j}=\tilde{\alpha}_{j i} \in \mathcal{C}^{1}\left(\overline{\mathfrak{D}}_{\varrho}\right), \quad \tilde{\alpha}_{i} \in \mathcal{C}\left(\overline{\mathfrak{D}}_{\varrho}\right), \quad \tilde{\alpha}_{0}, \widetilde{W} \in L_{\infty}\left(\mathfrak{O}_{\varrho}\right), \quad 1 \leqslant i, j \leqslant N,
$$

be any regular extensions from $\bar{\Omega}$ to $\overline{\mathfrak{D}}_{\varrho}$ of each of the coefficients

$$
\alpha_{i j}=\alpha_{j i}, \quad \alpha_{i}, \quad \alpha_{0}, \quad W, \quad 1 \leqslant i, j \leqslant N,
$$

respectively, and consider the auxiliary differential operator

$$
\tilde{\mathfrak{L}}:=-\sum_{i, j=1}^{N} \tilde{\alpha}_{i j} \frac{\partial^{2}}{\partial x_{i} \partial x_{j}}+\sum_{i=1}^{N} \tilde{\alpha}_{i} \frac{\partial}{\partial x_{i}}+\tilde{\alpha}_{0} \quad \text { in } \mathfrak{O}_{\varrho_{1}} .
$$

Since $\mathfrak{L}$ is strongly uniformly elliptic in $\Omega$ with ellipticity constant $\mu>0, \varrho \in\left(0, \varrho_{1}\right)$ exists for which the corresponding $\widetilde{\mathfrak{L}}$ is strongly uniformly elliptic in

$$
\widetilde{\Omega}:=\mathfrak{O}_{\varrho}
$$

with ellipticity constant $\mu / 2$. Now, we will consider the auxiliary potentials

$$
\tilde{\mathcal{X}}:=\left\{\begin{array}{ll}
1 & \text { in } \widetilde{\Omega} \backslash \Omega, \\
\mathcal{X} & \text { in } \Omega,
\end{array} \quad \widetilde{V}:= \begin{cases}1 & \text { in } \widetilde{\Omega} \backslash \Omega, \\
V & \text { in } \Omega,\end{cases}\right.
$$

the boundary operator

$$
\widetilde{\mathfrak{B}}(b):= \begin{cases}\mathfrak{D} & \text { on } \partial \widetilde{\Omega} \backslash \Gamma_{1}, \\ \partial_{\nu}+b & \text { on } \Gamma_{1},\end{cases}
$$

and any regular extension of $f$, say

$$
\tilde{f} \in \mathcal{C}^{1}(\overline{\widetilde{\Omega}} \times[0, \infty), \mathbb{R}),
$$

from $\bar{\Omega} \times[0, \infty)$ to $\overline{\widetilde{\Omega}} \times[0, \infty)$, such that

$$
\lim _{u \nearrow \infty} \tilde{f}(x, u)=\infty \quad \text { uniformly in } \overline{\widetilde{\Omega}} .
$$

Since $\mathcal{X}, V \in \mathcal{A}_{\Gamma_{0}, \Gamma_{1}}(\Omega)$, it is easy to see that

$$
\widetilde{\mathcal{X}}, \widetilde{V} \in \mathcal{A}_{\partial \widetilde{\Omega} \backslash \Gamma_{1}, \Gamma_{1}}(\widetilde{\Omega}) .
$$


Moreover, by construction,

$$
\bigcup_{j=1}^{q} \Gamma_{0}^{i_{j}} \subset \widetilde{\Omega}
$$

and, since $\widetilde{\mathcal{X}}=\widetilde{V}=1$ in $\widetilde{\Omega} \backslash \Omega$,

$$
\widetilde{\Omega}_{\widetilde{\mathcal{X}}}^{0}=\Omega_{\mathcal{X}}^{0}, \quad \widetilde{\Omega}_{\widetilde{V}}^{0}=\Omega_{V}^{0} \subset \bar{\Omega}, \quad \widetilde{K}_{\widetilde{V}}=K_{V} \subset \widetilde{\Omega} .
$$

Thus, thanks to (1.7),

$$
\left(\partial \widetilde{\Omega} \backslash \Gamma_{1}\right) \cap\left(\partial \widetilde{\Omega}_{\widetilde{V}}^{0} \cup \widetilde{K}_{\widetilde{V}}\right)=\left(\partial \widetilde{\Omega} \backslash \Gamma_{1}\right) \cap\left(\partial \Omega_{V}^{0} \cup K_{V}\right)=\left(\partial \widetilde{\Omega} \backslash \Gamma_{1}\right) \cap K_{V}
$$

and, hence, it follows from the construction of $\Gamma_{0}^{i_{j}}, 1 \leqslant j \leqslant q$, and $\widetilde{\Omega}$ that

$$
\left(\partial \widetilde{\Omega} \backslash \Gamma_{1}\right) \cap\left(\partial \widetilde{\Omega}_{\widetilde{V}}^{0} \cup \widetilde{K}_{\widetilde{V}}\right)=\emptyset .
$$

Moreover, thanks to (3.30), condition (2.8) is satisfied on

$$
\Gamma_{1} \cap \partial \widetilde{\Omega}_{\widetilde{V}}^{0}=\Gamma_{1} \cap \partial \Omega_{V}^{0}
$$

and, due to (1.7),

$$
\widetilde{\mathfrak{B}}\left(b, \widetilde{\Omega}_{V}^{0}\right)=\widetilde{\mathfrak{B}}\left(b, \Omega_{V}^{0}\right)=\mathfrak{B}\left(b, \Omega_{V}^{0}\right) .
$$

Thus,

$$
\lambda \in \Lambda\left[0, \Omega_{V}^{0}, \mathfrak{B}\left(b, \Omega_{V}^{0}\right)\right]=\Lambda\left[0, \widetilde{\Omega}_{\widetilde{V}}^{0}, \widetilde{\mathfrak{B}}\left(b, \widetilde{\Omega}_{\widetilde{V}}^{0}\right)\right]
$$

and, thanks to (3.31), condition (3.5) is satisfied for the new problem in $\widetilde{\Omega}$. Therefore, we can apply the result of part (i) in the special case when (3.5) is satisfied to the extended problem

$$
\begin{cases}\widetilde{\mathfrak{L}} \tilde{u}+\gamma \widetilde{V}(x) \tilde{u}=\lambda \widetilde{W}(x) \tilde{u}-\widetilde{\mathcal{X}}(x) \tilde{f}(x, \tilde{u}) \tilde{u} & \text { in } \widetilde{\Omega}, \\ \widetilde{\mathfrak{B}}(b) \tilde{u}=0 & \text { on } \partial \widetilde{\Omega} .\end{cases}
$$

As a result, for each $\delta>0$ sufficiently small there exist $\tilde{\Lambda}(\delta)>0$ and a positive function

$$
\overline{\tilde{u}}_{\delta}: \overline{\widetilde{\Omega}} \rightarrow[0, \infty)
$$

such that

$$
\overline{\tilde{u}}_{\delta}(x)>0 \quad \text { for each } x \in \widetilde{\Omega},
$$

and

$$
\begin{cases}{\left[\widetilde{\mathfrak{L}}+\gamma \widetilde{V}-\lambda \widetilde{W}+\widetilde{\mathcal{X}} \tilde{f}\left(\cdot, \overline{\tilde{u}}_{\delta}\right)\right] \overline{\tilde{u}}_{\delta}>0} & \text { in } \widetilde{\Omega}, \\ \widetilde{\mathfrak{B}}(b) \overline{\tilde{u}}_{\delta}=0 & \text { on } \partial \widetilde{\Omega}\end{cases}
$$

for each $\gamma>\tilde{\Lambda}(\delta)$. Now, set

$$
\bar{u}_{\delta}:=\left.\overline{\tilde{u}}_{\delta}\right|_{\Omega} .
$$


Then, thanks to (3.33) and (3.34), for each $\gamma>\tilde{\Lambda}(\delta)$ we have that

$$
\left[\mathfrak{L}+\gamma V-\lambda W+\mathcal{X} f\left(\cdot, \bar{u}_{\delta}\right)\right] \bar{u}_{\delta}=\left[\widetilde{\mathfrak{L}}+\gamma \widetilde{V}-\lambda \widetilde{W}+\widetilde{\mathcal{X}} \tilde{f}\left(\cdot, \overline{\tilde{u}}_{\delta}\right)\right] \overline{\tilde{u}}_{\delta} \geqslant 0 \quad \text { in } \Omega .
$$

Moreover, since $\bigcup_{j=1}^{q} \Gamma_{0}^{i_{j}} \subset \widetilde{\Omega}$,

$$
\bar{u}_{\delta}(x)=\overline{\tilde{u}}_{\delta}(x)>0 \quad \text { for each } x \in \bigcup_{j=1}^{q} \Gamma_{0}^{i_{j}} .
$$

Also,

$$
\bar{u}_{\delta}=\overline{\tilde{u}}_{\delta}=0 \quad \text { on } \Gamma_{0} \backslash \bigcup_{j=1}^{q} \Gamma_{0}^{i_{j}}
$$

and

$$
\left(\partial_{v}+b\right) \bar{u}_{\delta}=\left(\partial_{v}+b\right) \overline{\tilde{u}}_{\delta}=0 \quad \text { on } \Gamma_{1} .
$$

Thus,

$$
\mathfrak{B}(b) \bar{u}_{\delta}>0 \quad \text { on } \partial \Omega
$$

and, therefore, thanks to (3.35), for each $\delta>0$ sufficiently small the function $\bar{u}_{\delta}$ defined by (3.34), provides us with a positive strict supersolution of $P[\gamma, \lambda, \Omega, \mathfrak{B}(b)]$ if $\gamma>\tilde{\Lambda}(\delta)>0$. This completes the proof of Part (i).

Now, we shall prove part (ii). As in proving part (i) we will proceed separately distinguishing between the cases when (3.5) or (3.29) is satisfied.

Suppose (3.5). Then, (3.22) is satisfied. For each $\delta \in\left[0, \delta_{4}\right)$, let

$$
u_{\delta}:=u_{\left[\mathfrak{L}, \lambda W, \mathcal{X}, \Omega_{V}^{\delta}, \mathfrak{B}\left(b, \Omega_{V}^{\delta}\right)\right]}
$$

denote the unique positive solution of $P\left[0, \lambda, \Omega_{V}^{\delta}, \mathfrak{B}\left(b, \Omega_{V}^{\delta}\right)\right]$. Since (2.8) holds on $\Gamma_{1} \cap \partial \Omega_{V}^{0}$, it follows from Theorem 2.19 that

$$
\lim _{\delta \searrow 0}\left\|u_{\delta}-u_{0}\right\|_{H^{1}\left(\Omega_{V}^{0}\right)}=0 .
$$

Moreover, the positive strict supersolution $\bar{u}_{\delta}$ defined through (3.25) satisfies

$$
\left.\bar{u}_{\delta}\right|_{\Omega_{V}^{0}}=\left.u_{\delta}\right|_{\Omega_{V}^{0}},
$$

by definition, and, therefore, (3.37) implies

$$
\lim _{\delta \searrow 0}\left\|\bar{u}_{\delta}-u_{0}\right\|_{H^{1}\left(\Omega_{V}^{0}\right)}=0 .
$$

This shows (3.2).

We now prove (3.3). By definition of $\bar{u}_{\delta}$, it follows from (3.23) and (3.24) that, for each $\delta>0$ sufficiently small,

$$
\left\|\bar{u}_{\delta}\right\|_{L_{\infty}\left(H_{\delta / 2}^{\eta}\right)} \leqslant \delta .
$$


Let $K$ be a compact subset of $\bar{\Omega} \backslash \bar{\Omega}_{V}^{0}$. By construction, there exists

$$
\delta_{5}:=\delta_{5}(K) \in\left(0, \delta_{4}\right)
$$

such that, for each $\delta \in\left(0, \delta_{5}\right)$,

$$
K \subset \bar{\Omega} \backslash \bar{\Omega}_{V}^{\delta / 2}
$$

Thus, it follows from (3.38) and (3.39) that

$$
\left\|\bar{u}_{\delta}\right\|_{L_{\infty}\left(K \cap H_{\delta / 2}^{\eta}\right)} \leqslant \delta, \quad\left\|\bar{u}_{\delta}\right\|_{L_{\infty}\left(K \backslash H_{\delta / 2}^{\eta}\right)}=\left\|\zeta_{\delta}\right\|_{L_{\infty}\left(K \backslash H_{\delta / 2}^{\eta}\right)},
$$

since, by definition, $\bar{u}_{\delta}=\zeta_{\delta}$ on $K \backslash H_{\delta / 2}^{\eta}$, and, hence,

$$
\left\|\bar{u}_{\delta}\right\|_{L_{\infty}(K)} \leqslant \max \left\{\delta,\left\|\zeta_{\delta}\right\|_{L_{\infty}\left(K \backslash H_{\delta / 2}^{\eta}\right)}\right\} .
$$

Finally, since $\zeta_{\delta}$ is an arbitrary regular positive extension of

$$
u_{\delta} \cup \bigcup_{j=1}^{\ell_{V}(\eta)} \delta \vartheta_{\delta}^{j} \cup \bigcup_{j=1}^{p} \delta \psi_{\delta}^{i_{j}} \cup \bigcup_{j=1}^{n_{0}} \delta \xi_{\delta}^{j}
$$

from

$$
\bar{\Omega}_{V}^{\delta / 2} \cup \bigcup_{j=1}^{\ell_{V}(\eta)} \bar{G}_{j}^{\eta} \cup \bigcup_{j=1}^{p} \overline{\mathcal{N}}_{\delta / 2}^{1, i_{j}} \cup \bigcup_{j=1}^{n_{0}} \overline{\mathcal{N}}_{\delta / 2}^{0, j}
$$

to $\bar{\Omega}$, and (3.23), (3.24) imply

$$
\lim _{\delta \searrow 0}\left\|\delta \vartheta_{\delta}^{j}\right\|_{L_{\infty}\left(G_{j}^{\eta}\right)}=\lim _{\delta \searrow 0}\left\|\delta \psi_{\delta}^{i_{j}}\right\|_{L_{\infty}\left(\mathcal{N}_{\delta / 2}^{1, i_{j}}\right)}=\lim _{\delta \searrow 0}\left\|\delta \xi_{\delta}^{j}\right\|_{L_{\infty}\left(\mathcal{N}_{\delta / 2}^{0, j}\right)}=0
$$

passing to the limit as $\delta \searrow 0$ in (3.40) it is rather clear that $\zeta_{\delta}$ can be adjusted so that (3.3) holds; (3.4) is easily obtained from (3.2) and (3.3). This completes the proof of part (ii) under (3.5).

Now, suppose (3.29), instead of (3.5). Then, arguing as in the proof of part (i) under condition (3.29), we have that the positive strict supersolution $\overline{\tilde{u}}_{\delta}$ built up in $\widetilde{\Omega}$ satisfies

$$
\lim _{\delta \searrow 0}\left\|\overline{\tilde{u}}_{\delta}-u_{\left[\widetilde{\mathfrak{L}}, \lambda \widetilde{W}, \widetilde{\mathcal{X}}, \widetilde{\Omega}_{\tilde{V}}^{0}, \widetilde{\mathfrak{B}}\left(b, \widetilde{\Omega}_{\tilde{V}}^{0}\right)\right]}\right\|_{H^{1}\left(\widetilde{\Omega}_{\tilde{V}}^{0}\right)}=0 .
$$

On the other hand, by construction, we have that

$$
\widetilde{\Omega}_{\widetilde{V}}^{0}=\Omega_{V}^{0} \subset \bar{\Omega}
$$

and

$$
\left.\overline{\tilde{u}}_{\delta}\right|_{\widetilde{\Omega}_{\tilde{V}}^{0}}=\bar{u}_{\delta}, \quad \widetilde{\mathfrak{B}}\left(b, \widetilde{\Omega}_{\tilde{V}}^{0}\right)=\widetilde{\mathfrak{B}}\left(b, \Omega_{V}^{0}\right)=\mathfrak{B}\left(b, \Omega_{V}^{0}\right),
$$


S. CANO-CASANOVA, J. LÓPEZ-GÓMEZ / Ann. I. H. Poincaré - AN 20 (2003) 999-1041 1027

where $\bar{u}_{\delta}$ is the positive strict supersolution defined by (3.25). Moreover,

$$
\left.\widetilde{\mathfrak{L}}\right|_{\widetilde{\Omega}_{\tilde{V}}^{0}}=\mathfrak{L},\left.\quad \widetilde{W}\right|_{\widetilde{\Omega}_{\tilde{V}}^{0}}=W,\left.\quad \widetilde{\mathcal{X}}\right|_{\widetilde{\Omega}_{\tilde{V}}^{0}}=\mathcal{X} .
$$

Thus, (3.41) becomes into

$$
\lim _{\delta \searrow 0}\left\|\bar{u}_{\delta}-u_{\left[\mathfrak{L}, \lambda W, \mathcal{X}, \Omega_{V}^{0}, \mathfrak{B}\left(b, \Omega_{V}^{0}\right)\right]}\right\|_{H^{1}\left(\Omega_{V}^{0}\right)}=0,
$$

so proving (3.2) under condition (3.29).

Similarly, adapting the argument given in the case when condition (3.5) is satisfied, we have that

$$
\lim _{\delta \searrow 0}\left\|\overline{\tilde{u}}_{\delta}\right\|_{L_{\infty}(\widetilde{K})}=0
$$

in any compact subset

$$
\widetilde{K} \subset \overline{\widetilde{\Omega}} \backslash \overline{\widetilde{\Omega}}_{\widetilde{V}}^{0}
$$

In particular, (3.42) holds in any compact subset $K$ of $\bar{\Omega} \backslash \bar{\Omega}_{V}^{0}$, since

$$
\bar{\Omega} \backslash \bar{\Omega}_{V}^{0}=\bar{\Omega} \backslash \overline{\widetilde{\Omega}}_{V}^{0} \subset \overline{\widetilde{\Omega}} \backslash \overline{\widetilde{\Omega}}_{\widetilde{V}}^{0} .
$$

Therefore, since

$$
\left.\overline{\tilde{u}}_{\delta}\right|_{\bar{\Omega} \backslash \bar{\Omega}_{V}^{0}}=\bar{u}_{\delta},
$$

(3.42) implies (3.3); (3.4) follows readily from (3.2) and (3.3). This completes the proof of part (ii) and concludes the proof of the proposition.

Now, we are ready to prove Theorem 1.3.

Proof of Theorem 1.3. - Suppose $\gamma_{0}>0$ exists such that

$$
\lambda \in \Lambda\left[0, \Omega_{V}^{0}, \mathfrak{B}\left(b, \Omega_{V}^{0}\right)\right] \cap \Lambda[\gamma, \Omega, \mathfrak{B}(b)]
$$

for each $\gamma>\gamma_{0}$, and (2.8) holds on $\Gamma_{1} \cap \partial \Omega_{V}^{0}$. For each $\gamma>\gamma_{0}$, let

$$
u_{[\mathfrak{L}+\gamma V, \lambda W, \mathcal{X}, \Omega, \mathfrak{B}(b)]} \wedge \quad u_{\left[\mathfrak{L}, \lambda W, \mathcal{X}, \Omega_{V}^{0}, \mathfrak{B}\left(b, \Omega_{V}^{0}\right)\right]}
$$

denote the unique positive solutions of problems $P[\gamma, \lambda, \Omega, \mathfrak{B}(b)]$ and $P\left[0, \lambda, \Omega_{V}^{0}\right.$, $\left.\mathfrak{B}\left(b, \Omega_{V}^{0}\right)\right]$, respectively. Since $u_{[\mathfrak{L}+\gamma V, \lambda W, \mathcal{X}, \Omega, \mathfrak{B}(b)]}$ is strongly positive in $\Omega$, we have that

$$
\mathfrak{B}\left(b, \Omega_{V}^{0}\right) u_{[\mathfrak{L}+\gamma V, \lambda W, \mathcal{X}, \Omega, \mathfrak{B}(b)]}=u_{[\mathfrak{L}+\gamma V, \lambda W, \mathcal{X}, \Omega, \mathfrak{B}(b)]}>0 \quad \text { on } \partial \Omega_{V}^{0} \cap \Omega .
$$

Thus, $u_{[\mathfrak{L}+\gamma V, \lambda W, \mathcal{X}, \Omega, \mathfrak{B}(b)]}$ is a positive strict supersolution of $P\left[0, \lambda, \Omega_{V}^{0}, \mathfrak{B}\left(b, \Omega_{V}^{0}\right)\right]$ for each $\gamma>\gamma_{0}$, and, hence, thanks to Theorem 2.16,

$$
u_{\left[\mathfrak{L}, \lambda W, \mathcal{X}, \Omega_{V}^{0}, \mathfrak{B}\left(b, \Omega_{V}^{0}\right)\right]}<u_{[\mathfrak{L}+\gamma V, \lambda W, \mathcal{X}, \Omega, \mathfrak{B}(b)]} \text { in } \Omega_{V}^{0} \text { for each } \gamma>\gamma_{0} .
$$


Therefore, the auxiliary function

$$
u_{*}:= \begin{cases}u_{\left[\mathcal{L}, \lambda W, \mathcal{X}, \Omega_{V}^{0}, \mathfrak{B}\left(b, \Omega_{V}^{0}\right)\right]} & \text { in } \bar{\Omega}_{V}^{0}, \\ 0 & \text { in } \bar{\Omega} \backslash \bar{\Omega}_{V}^{0}\end{cases}
$$

satisfies

$$
u_{*}<u_{[\mathfrak{L}+\gamma V, \lambda W, \mathcal{X}, \Omega, \mathfrak{B}(b)]} \text { in } \Omega \text { for each } \gamma>\gamma_{0} .
$$

On the other hand, since we are working under the assumptions of Proposition 3.1, $\delta_{0}>0$ exists such that for each $\delta \in\left(0, \delta_{0}\right)$ there are a real number $\Lambda(\delta)>0$ and a positive function $\bar{u}_{\delta}$ such that $\bar{u}_{\delta}$ is a positive strict supersolution of $P[\gamma, \lambda, \Omega, \mathfrak{B}(b)]$ for each $\gamma>\Lambda(\delta)$

$$
\lim _{\delta \searrow 0}\left\|\bar{u}_{\delta}-u_{\left[\mathcal{L}, \lambda W, \mathcal{X}, \Omega_{V}^{0}, \mathfrak{B}\left(b, \Omega_{V}^{0}\right)\right]}\right\|_{H^{1}\left(\Omega_{V}^{0}\right)}=0
$$

and, for any compact subset $K \subset \bar{\Omega} \backslash \bar{\Omega}_{V}^{0}$,

$$
\lim _{\delta \searrow 0}\left\|\bar{u}_{\delta}\right\|_{L_{\infty}(K)}=0 .
$$

In particular,

$$
\lim _{\delta \searrow 0} \bar{u}_{\delta}=\left\{\begin{array}{ll}
u_{\left[\mathfrak{L}, \lambda W, \mathcal{X}, \Omega_{V}^{0}, \mathfrak{B}\left(b, \Omega_{V}^{0}\right)\right]} & \text { in } \Omega_{V}^{0}, \\
0 & \text { in } \Omega \backslash \Omega_{V}^{0},
\end{array} \quad \text { a.e. in } \Omega .\right.
$$

Thanks to Theorem 2.16,

$$
u_{[\mathfrak{L}+\gamma V, \lambda W, \mathcal{X}, \Omega, \mathfrak{B}(b)]} \leqslant \bar{u}_{\delta} \quad \text { in } \Omega \text { for each } \delta \in\left(0, \delta_{0}\right) \text { and } \gamma>\Lambda(\delta) .
$$

Therefore, it follows from (3.45) and (3.46) that

$$
u_{*}<u_{[\mathfrak{L}+\gamma V, \lambda W, \mathcal{X}, \Omega, \mathfrak{B}(b)]} \leqslant \bar{u}_{\delta} \quad \text { in } \Omega \text { for each } \delta \in\left(0, \delta_{0}\right) \text { and } \gamma>\gamma_{\delta},
$$

where

$$
\gamma_{\delta}:=\max \left\{\Lambda(\delta), \gamma_{0}\right\} .
$$

Thanks to (3.47), for each $\delta \in\left(0, \delta_{0}\right)$ and $\gamma>\gamma_{\delta}$, we have that

$$
\left.u_{*}\right|_{\Omega_{V}^{0}}=u_{\left[\mathfrak{L}, \lambda W, \mathcal{X}, \Omega_{V}^{0}, \mathfrak{B}\left(b, \Omega_{V}^{0}\right)\right]} \leqslant u_{[\mathfrak{L}+\gamma V, \lambda W, \mathcal{X}, \Omega, \mathfrak{B}(b)]} \leqslant \bar{u}_{\delta} \quad \text { in } \Omega_{V}^{0}
$$

and, hence,

$$
\begin{aligned}
& \left\|u_{[\mathfrak{L}+\gamma V, \lambda W, \mathcal{X}, \Omega, \mathfrak{B}(b)]}-u_{\left[\mathfrak{L}, \lambda W, \mathcal{X}, \Omega_{V}^{0}, \mathfrak{B}\left(b, \Omega_{V}^{0}\right)\right]}\right\|_{L_{2}\left(\Omega_{V}^{0}\right)} \\
& \quad \leqslant\left\|\bar{u}_{\delta}-u_{\left[\mathfrak{L}, \lambda W, \mathcal{X}, \Omega_{V}^{0}, \mathfrak{B}\left(b, \Omega_{V}^{0}\right)\right]}\right\|_{L_{2}\left(\Omega_{V}^{0}\right)} .
\end{aligned}
$$

Thus, for each $\delta \in\left(0, \delta_{0}\right)$, we have that 
S. CANO-CASANOVA, J. LÓPEZ-GÓMEZ / Ann. I. H. Poincaré - AN 20 (2003) 999-1041 1029

$$
\begin{aligned}
& \underset{\gamma \nearrow \infty}{\limsup }\left\|u_{[\mathfrak{L}+\gamma V, \lambda W, \mathcal{X}, \Omega, \mathfrak{B}(b)]}-u_{\left[\mathcal{L}, \lambda W, \mathcal{X}, \Omega_{V}^{0}, \mathfrak{B}\left(b, \Omega_{V}^{0}\right)\right]}\right\|_{L_{2}\left(\Omega_{V}^{0}\right)} \\
& \quad \leqslant\left\|\bar{u}_{\delta}-u_{\left[\mathfrak{L}, \lambda W, \mathcal{X}, \Omega_{V}^{0}, \mathfrak{B}\left(b, \Omega_{V}^{0}\right)\right]}\right\|_{L_{2}\left(\Omega_{V}^{0}\right)} .
\end{aligned}
$$

On the other hand, thanks to Proposition 3.1, we already know that

$$
\lim _{\delta \searrow 0}\left\|\bar{u}_{\delta}-u_{\left[\mathfrak{L}, \lambda W, \mathcal{X}, \Omega_{V}^{0}, \mathfrak{B}\left(b, \Omega_{V}^{0}\right)\right]}\right\|_{L_{2}\left(\Omega_{V}^{0}\right)}=0,
$$

and, therefore, combining (3.50) and (3.51) gives

$$
\lim _{\gamma>\infty}\left\|u_{[\mathfrak{L}+\gamma V, \lambda W, \mathcal{X}, \Omega, \mathfrak{B}(b)]}-u_{\left[\mathfrak{L}, \lambda W, \mathcal{X}, \Omega_{V}^{0}, \mathfrak{B}\left(b, \Omega_{V}^{0}\right)\right]}\right\|_{L_{2}\left(\Omega_{V}^{0}\right)}=0 .
$$

Now, fix $\delta \in\left(0, \delta_{0}\right)$. Then, thanks to (3.48),

$$
\left\|u_{[\mathfrak{L}+\gamma V, \lambda W, \mathcal{X}, \Omega, \mathfrak{B}(b)]}\right\|_{L_{\infty}\left(\Omega_{V}^{0}\right)} \leqslant\left\|\bar{u}_{\delta}\right\|_{L_{\infty}\left(\Omega_{V}^{0}\right)} \quad \text { for each } \gamma>\gamma_{\delta}
$$

and, hence, there exists a constant $C>0$ such that

$$
\left\|u_{[\mathcal{L}+\gamma V, \lambda W, \mathcal{X}, \Omega, \mathfrak{B}(b)]}-u_{\left[\mathfrak{L}, \lambda W, \mathcal{X}, \Omega_{V}^{0}, \mathfrak{B}\left(b, \Omega_{V}^{0}\right)\right]}\right\|_{L_{\infty}\left(\Omega_{V}^{0}\right)} \leqslant C \quad \text { for each } \gamma>\gamma_{\delta} .
$$

Thus, (3.52) implies

$$
\lim _{\gamma \nearrow \infty}\left\|u_{[\mathfrak{L}+\gamma V, \lambda W, \mathcal{X}, \Omega, \mathfrak{B}(b)]}-u_{\left[\mathfrak{L}, \lambda W, \mathcal{X}, \Omega_{V}^{0}, \mathfrak{B}\left(b, \Omega_{V}^{0}\right)\right]}\right\|_{L_{p}\left(\Omega_{V}^{0}\right)}=0 \quad \text { for each } p \in[2, \infty) .
$$

On the other hand, since $L_{2}\left(\Omega_{V}^{0}\right) \hookrightarrow L_{p}\left(\Omega_{V}^{0}\right)$ if $p \in[1,2)$, (3.52) gives

$$
\lim _{\gamma \nearrow \infty}\left\|u_{[\mathfrak{L}+\gamma V, \lambda W, \mathcal{X}, \Omega, \mathfrak{B}(b)]}-u_{\left[\mathfrak{L}, \lambda W, \mathcal{X}, \Omega_{V}^{0}, \mathfrak{B}\left(b, \Omega_{V}^{0}\right)\right]}\right\|_{L_{p}\left(\Omega_{V}^{0}\right)}=0 \quad \text { for each } p \in[1,2) .
$$

This concludes the proof of (1.9).

Let $K$ be a compact subset of $\bar{\Omega} \backslash \bar{\Omega}_{V}^{0}$. Then, thanks to (3.47),

$$
0=\left.u_{*}\right|_{K} \leqslant\left. u_{[\mathfrak{L}+\gamma V, \lambda W, \mathcal{X}, \Omega, \mathfrak{B}(b)]}\right|_{K} \leqslant\left.\bar{u}_{\delta}\right|_{K} \quad \text { for each } \delta \in\left(0, \delta_{0}\right) \text { and } \gamma>\gamma_{\delta},
$$

and, hence,

$$
\left\|u_{[\mathfrak{L}+\gamma V, \lambda W, \mathcal{X}, \Omega, \mathfrak{B}(b)]}\right\|_{L_{\infty}(K)} \leqslant\left\|\bar{u}_{\delta}\right\|_{L_{\infty}(K)} \quad \text { for each } \delta \in\left(0, \delta_{0}\right) \text { and } \gamma>\gamma_{\delta} .
$$

Thus, passing to the limit as $\gamma \nearrow \infty$ in (3.54) gives

$$
0 \leqslant \limsup _{\gamma / \infty}\left\|u_{[\mathfrak{L}+\gamma V, \lambda W, \mathcal{X}, \Omega, \mathfrak{B}(b)]}\right\|_{L_{\infty}(K)} \leqslant\left\|\bar{u}_{\delta}\right\|_{L_{\infty}(K)} \quad \text { for each } \delta \in\left(0, \delta_{0}\right) .
$$

On the other hand, thanks to Proposition 3.1, we have that

$$
\lim _{\delta \searrow 0}\left\|\bar{u}_{\delta}\right\|_{L_{\infty}(K)}=0
$$


1030 S. CANO-CASANOVA, J. LÓPEZ-GÓMEZ / Ann. I. H. Poincaré - AN 20 (2003) 999-1041

and, therefore, (3.55) implies

$$
\lim _{\gamma \nearrow \infty}\left\|u_{[\mathfrak{L}+\gamma V, \lambda W, \mathcal{X}, \Omega, \mathfrak{B}(b)]}\right\|_{L_{\infty}(K)}=0 .
$$

This completes the proof of (1.10) and concludes the proof of the theorem.

\section{Some sufficient conditions so that $1 \Rightarrow 2$ in Theorem 1.3}

The following results provide us with some sufficient conditions ensuring that $\lambda \in$ $\Lambda[\gamma, \Omega, \mathfrak{B}(b)]$ for any $\gamma$ sufficiently large whenever $\lambda \in \Lambda\left[0, \Omega_{V}^{0}, \mathfrak{B}\left(b, \Omega_{V}^{0}\right)\right]$.

THEOREM 4.1. - Suppose $\lambda \in \Lambda\left[0, \Omega_{V}^{0}, \mathfrak{B}\left(b, \Omega_{V}^{0}\right)\right]$, (2.8) holds on $\Gamma_{1} \cap\left(\partial \Omega_{V}^{0} \cup\right.$ $\left.\partial \Omega_{\mathcal{X}}^{0}\right)$ and

$$
\begin{aligned}
& \mathcal{X} \in \mathcal{A}_{\partial \Omega_{V}^{0} \backslash \Gamma_{1}, \Gamma_{1} \cap \partial \Omega_{V}^{0}}\left(\Omega_{V}^{0}\right) \wedge V \in \mathcal{A}_{\partial \Omega_{\mathcal{X}}^{0} \backslash \Gamma_{1}, \Gamma_{1} \cap \partial \Omega_{\mathcal{X}}^{0}}\left(\Omega_{\mathcal{X}}^{0}\right) \wedge \Omega_{V}^{0} \cap \Omega_{\mathcal{X}}^{0} \in \mathcal{C}^{2}, \\
& \text { if } \Omega_{V}^{0} \cap \Omega_{\mathcal{X}}^{0} \neq \emptyset \text {, or } \\
& \quad \mathcal{X} \in \mathcal{A}_{\partial \Omega_{V}^{0} \backslash \Gamma_{1}, \Gamma_{1} \cap \partial \Omega_{V}^{0}}^{+}\left(\Omega_{V}^{0}\right) \wedge V \in \mathcal{A}_{\partial \Omega_{\mathcal{X}}^{0} \backslash \Gamma_{1}, \Gamma_{1} \cap \partial \Omega_{\mathcal{X}}^{0}}^{+}\left(\Omega_{\mathcal{X}}^{0}\right),
\end{aligned}
$$

when $\Omega_{V}^{0} \cap \Omega_{\mathcal{X}}^{0}=\emptyset$. Then, there exists $\gamma_{0}>0$ such that

$$
\lambda \in \bigcap_{\gamma \geqslant \gamma_{0}} \Lambda[\gamma, \Omega, \mathfrak{B}(b)]
$$

and, therefore, Theorem 1.3 can be applied.

Proof. - Suppose

$$
\left(\left[\Omega_{V}^{0}\right]_{\mathcal{X}}^{0}=\left[\Omega_{\mathcal{X}}^{0}\right]_{V}^{0}=\right) \Omega_{V}^{0} \cap \Omega_{\mathcal{X}}^{0} \neq \emptyset .
$$

Then, since $\Omega_{V}^{0} \cap \Omega_{\mathcal{X}}^{0}$ is of class $\mathcal{C}^{2},(2.8)$ holds on $\Gamma_{1} \cap\left(\partial \Omega_{V}^{0} \cup \partial \Omega_{\mathcal{X}}^{0}\right)$, and

$$
\partial\left(\Omega_{V}^{0} \cap \Omega_{\mathcal{X}}^{0}\right) \subset \partial \Omega_{V}^{0} \cup \partial \Omega_{\mathcal{X}}^{0}
$$

necessarily (2.8) holds on $\Gamma_{1} \cap \partial\left(\Omega_{V}^{0} \cap \Omega_{\mathcal{X}}^{0}\right)$. Thus, thanks to Theorem 2.12,

$$
\lambda \in \Lambda\left[0, \Omega_{V}^{0}, \mathfrak{B}\left(b, \Omega_{V}^{0}\right)\right]
$$

$$
\text { 企 }
$$

$$
\sigma\left[\mathfrak{L}-\lambda W+\mathcal{X} f(\cdot, 0), \Omega_{V}^{0}, \mathfrak{B}\left(b, \Omega_{V}^{0}\right)\right]<0<\sigma\left[\mathfrak{L}-\lambda W,\left[\Omega_{V}^{0}\right]_{\mathcal{X}}^{0}, \mathfrak{B}\left(b,\left[\Omega_{V}^{0}\right]_{\mathcal{X}}^{0}\right)\right] .
$$

Similarly, for any $\gamma>0$,

$$
\begin{gathered}
\lambda \in \Lambda[\gamma, \Omega, \mathfrak{B}(b)] \\
\text { 步 } \\
\sigma[\mathfrak{L}+\gamma V-\lambda W+\mathcal{X} f(\cdot, 0), \Omega, \mathfrak{B}(b)]<0<\sigma\left[\mathfrak{L}+\gamma V-\lambda W, \Omega_{\mathcal{X}}^{0}, \mathfrak{B}\left(b, \Omega_{\mathcal{X}}^{0}\right)\right] .
\end{gathered}
$$


S. CANO-CASANOVA, J. LÓPEZ-GÓMEZ / Ann. I. H. Poincaré - AN 20 (2003) 999-1041 1031

On the other hand, since (2.8) holds on $\Gamma_{1} \cap \partial\left[\Omega_{\mathcal{X}}^{0}\right]_{V}^{0}$, it follows from Theorem 2.9 that

$$
\lim _{\gamma>\infty} \sigma\left[\mathfrak{L}+\gamma V-\lambda W, \Omega_{\mathcal{X}}^{0}, \mathfrak{B}\left(b, \Omega_{\mathcal{X}}^{0}\right)\right]=\sigma\left[\mathfrak{L}-\lambda W,\left[\Omega_{\mathcal{X}}^{0}\right]_{V}^{0}, \mathfrak{B}\left(b,\left[\Omega_{\mathcal{X}}^{0}\right]_{V}^{0}\right)\right] .
$$

Similarly,

$$
\begin{array}{r}
\lim _{\gamma>\infty} \sigma[\mathfrak{L}+\gamma V-\lambda W+\mathcal{X} f(\cdot, 0), \Omega, \mathfrak{B}(b)] \\
\quad=\sigma\left[\mathfrak{L}-\lambda W+\mathcal{X} f(\cdot, 0), \Omega_{V}^{0}, \mathfrak{B}\left(b, \Omega_{V}^{0}\right)\right] .
\end{array}
$$

Therefore, thanks to (4.2)-(4.4) and (4.5), condition (4.1) is satisfied for some $\gamma_{0}>0$ if $\lambda \in \Lambda\left[0, \Omega_{V}^{0}, \mathfrak{B}\left(b, \Omega_{V}^{0}\right)\right]$. This completes the proof of the theorem in this special case.

Now, suppose

$$
\left(\left[\Omega_{V}^{0}\right]_{\mathcal{X}}^{0}=\left[\Omega_{\mathcal{X}}^{0}\right]_{V}^{0}=\right) \Omega_{V}^{0} \cap \Omega_{\mathcal{X}}^{0}=\emptyset .
$$

Since

$$
\left[\Omega_{V}^{0}\right]_{\mathcal{X}}^{0}=\emptyset \wedge \mathcal{X} \in \mathcal{A}_{\partial \Omega_{V}^{0} \backslash \Gamma_{1}, \Gamma_{1} \cap \partial \Omega_{V}^{0}}\left(\Omega_{V}^{0}\right),
$$

it follows from Theorem 2.14 that

$$
\lambda \in \Lambda\left[0, \Omega_{V}^{0}, \mathfrak{B}\left(b, \Omega_{V}^{0}\right)\right] \Longleftrightarrow \sigma\left[\mathfrak{L}-\lambda W+\mathcal{X} f(\cdot, 0), \Omega_{V}^{0}, \mathfrak{B}\left(b, \Omega_{V}^{0}\right)\right]<0 .
$$

Similarly, thanks to Theorem 2.12, the equivalence (4.3) holds.

Since $V \in \mathcal{A}_{\Gamma_{0}, \Gamma_{1}}(\Omega)$ and (2.8) is satisfied on $\Gamma_{1} \cap \partial \Omega_{V}^{0}$, it follows from Theorem 2.9 that (4.5) holds. Thus, since $\lambda \in \Lambda\left[0, \Omega_{V}^{0}, \mathfrak{B}\left(b, \Omega_{V}^{0}\right)\right]$, it follows from (4.5) and (4.6) that

$$
\lim _{\gamma>\infty} \sigma[\mathfrak{L}+\gamma V-\lambda W+\mathcal{X} f(\cdot, 0), \Omega, \mathfrak{B}(b)]<0 .
$$

Similarly, since

it follows from Theorem 2.10 that

$$
V \in \mathcal{A}_{\partial \Omega_{\mathcal{X}}^{0} \backslash \Gamma_{1}, \Gamma_{1} \cap \partial \Omega_{\mathcal{X}}^{0}}\left(\Omega_{\mathcal{X}}^{0}\right) \wedge \quad\left[\Omega_{\mathcal{X}}^{0}\right]_{V}^{0}=\emptyset,
$$

$$
\lim _{\gamma \nearrow \infty} \sigma\left[\mathfrak{L}+\gamma V-\lambda W, \Omega_{\mathcal{X}}^{0}, \mathfrak{B}\left(b, \Omega_{\mathcal{X}}^{0}\right)\right]=\infty .
$$

Then, combining (4.7) and (4.8), we find that, for any $\gamma>0$ sufficiently large, the following estimate is satisfied

$$
\sigma[\mathfrak{L}+\gamma V-\lambda W+\mathcal{X} f(\cdot, 0), \Omega, \mathfrak{B}(b)]<0<\sigma\left[\mathfrak{L}+\gamma V-\lambda W, \Omega_{\mathcal{X}}^{0}, \mathfrak{B}\left(b, \Omega_{\mathcal{X}}^{0}\right)\right] .
$$

Therefore, it follows from (4.3) and (4.9) that (4.1) must be satisfied for some $\gamma_{0}>0$. This completes the proof of the theorem.

It should be noted that Corollary 2.18 provides us some sufficient conditions for having

$$
\mathcal{X} \in \mathcal{A}_{\partial \Omega_{V}^{0} \backslash \Gamma_{1}, \Gamma_{1} \cap \partial \Omega_{V}^{0}}\left(\Omega_{V}^{0}\right) \wedge V \in \mathcal{A}_{\partial \Omega_{\mathcal{X}}^{0} \backslash \Gamma_{1}, \Gamma_{1} \cap \partial \Omega_{\mathcal{X}}^{0}}\left(\Omega_{\mathcal{X}}^{0}\right)
$$


1032 S. CANO-CASANOVA, J. LÓPEZ-GÓMEZ / Ann. I. H. Poincaré - AN 20 (2003) 999-1041

in case $\Omega_{V}^{0} \cap \Omega_{\mathcal{X}}^{0} \neq \emptyset$, and

$$
\mathcal{X} \in \mathcal{A}_{\partial \Omega_{V}^{0} \backslash \Gamma_{1}, \Gamma_{1} \cap \partial \Omega_{V}^{0}}^{+}\left(\Omega_{V}^{0}\right) \wedge V \in \mathcal{A}_{\partial \Omega_{\mathcal{X}}^{0} \backslash \Gamma_{1}, \Gamma_{1} \cap \partial \Omega_{\mathcal{X}}^{0}}^{+}\left(\Omega_{\mathcal{X}}^{0}\right)
$$

when $\Omega_{V}^{0} \cap \Omega_{\mathcal{X}}^{0}=\emptyset$.

THEOREM 4.2. - Assume $\lambda \in \Lambda\left[0, \Omega_{V}^{0}, \mathfrak{B}\left(b, \Omega_{V}^{0}\right)\right], \mathcal{X} \in \mathcal{A}_{\Gamma_{0}, \Gamma_{1}}^{+}(\Omega)$, and (2.8) holds on $\Gamma_{1} \cap \partial \Omega_{V}^{0}$. Then, $\gamma_{0}>0$ exists for which condition (4.1) is satisfied. Therefore, Theorem 1.3 can be applied.

Proof. - Since $\mathcal{X} \in \mathcal{A}_{\Gamma_{0}, \Gamma_{1}}^{+}(\Omega)$, Corollary 2.18 implies

$$
\mathcal{X} \in \mathcal{A}_{\partial \Omega_{V}^{0} \backslash \Gamma_{1}, \partial \Omega_{V}^{0} \cap \Gamma_{1}}^{+}\left(\Omega_{V}^{0}\right)
$$

and, hence, thanks to Theorem 2.14,

$$
\Lambda\left[0, \Omega_{V}^{0}, \mathfrak{B}\left(b, \Omega_{V}^{0}\right)\right]=\left\{\lambda \in \mathbb{R}: \sigma\left[\mathfrak{L}-\lambda W+\mathcal{X} f(\cdot, 0), \Omega_{V}^{0}, \mathfrak{B}\left(b, \Omega_{V}^{0}\right)\right]<0\right\} .
$$

Similarly, for each $\gamma>0$, we have that

$$
\Lambda[\gamma, \Omega, \mathfrak{B}(b)]=\{\lambda \in \mathbb{R}: \sigma[\mathfrak{L}+\gamma V-\lambda W+\mathcal{X} f(\cdot, 0), \Omega, \mathfrak{B}(b)]<0\} .
$$

On the other hand, since $V \in \mathcal{A}_{\Gamma_{0}, \Gamma_{1}}(\Omega)$ and (2.8) holds on $\Gamma_{1} \cap \partial \Omega_{V}^{0}$, Theorem 2.9 gives

$$
\begin{array}{r}
\lim _{\gamma>\infty} \sigma[\mathfrak{L}+\gamma V-\lambda W+\mathcal{X} f(\cdot, 0), \Omega, \mathfrak{B}(b)] \\
\quad=\sigma\left[\mathfrak{L}-\lambda W+\mathcal{X} f(\cdot, 0), \Omega_{V}^{0}, \mathfrak{B}\left(b, \Omega_{V}^{0}\right)\right] .
\end{array}
$$

Thus, since $\lambda \in \Lambda\left[0, \Omega_{V}^{0}, \mathfrak{B}\left(b, \Omega_{V}^{0}\right)\right]$, we find from (4.10) and (4.12) that

$$
\sigma[\mathfrak{L}+\gamma V-\lambda W+\mathcal{X} f(\cdot, 0), \Omega, \mathfrak{B}(b)]<0
$$

for each $\gamma$ sufficiently large. Therefore, thanks to (4.11), $\lambda \in \Lambda[\gamma, \Omega, \mathfrak{B}(b)]$ for $\gamma$ large. This completes the proof.

It should be noted that if $V \in \mathcal{A}_{\Gamma_{0}, \Gamma_{1}}^{+}(\Omega)$, then it does not make sense analyzing the limiting behavior as $\gamma \nearrow \infty$ of the positive solution of (1.1). Indeed, in such case, Theorem 2.10 implies

$$
\lim _{\gamma>\infty} \sigma[\mathfrak{L}+\gamma V-\lambda W+\mathcal{X} f(\cdot, 0), \Omega, \mathfrak{B}(b)]=\infty
$$

and, thanks to Theorem 2.12 and Theorem 2.14, for each $\gamma>0$,

$$
\Lambda[\gamma, \Omega, \mathfrak{B}(b)] \subseteq\{\lambda \in \mathbb{R}: \sigma[\mathfrak{L}+\gamma V-\lambda W+\mathcal{X} f(\cdot, 0), \Omega, \mathfrak{B}(b)]<0\} .
$$

Therefore, there exists $\gamma_{0}:=\gamma_{0}(\lambda)>0$ such that

$$
\lambda \notin \bigcup_{\gamma \geqslant \gamma_{0}} \Lambda[\gamma, \Omega, \mathfrak{B}(b)] .
$$


It turns out that the positive solution of problem (1.1) for small values of $\gamma$ becomes the zero solution at the unique value of $\gamma$ for which

$$
\sigma[\mathfrak{L}+\gamma V-\lambda W+\mathcal{X} f(\cdot, 0), \Omega, \mathfrak{B}(b)]=0 .
$$

Therefore, it is consistent saying that the limiting profile of the maximal nonnegative solution is zero.

\section{Permanence under unlimited aggression is possible}

This section applies Theorem 1.3 for obtaining the following biological principle: no species can be driven to extinction by a competitor if it possesses a refuge and its birth rate in the overall habitat is sufficiently large. Moreover, it will be shown how the species concentrates within the refuge when it suffers high level aggressions. For establishing these principles we adopt as a model for competing species the following spatially heterogeneous evolutionary model of Lotka-Volterra type with diffusion and transport effects

$$
\begin{cases}\frac{\partial u}{\partial t}+\mathfrak{L}_{1} u=\lambda u-\mathcal{X}_{1}(x) u^{2}-\gamma_{1} V_{1}(x) u v & \text { in } \Omega \times(0, \infty), \\ \frac{\partial v}{\partial t}+\mathfrak{L}_{2} v=\mu v-\mathcal{X}_{2}(x) v^{2}-\gamma_{2} V_{2}(x) u v & \text { in } \Omega \times(0, \infty), \\ \mathfrak{B}_{1} u=\mathfrak{B}_{2} v=0 & \text { on } \partial \Omega \times(0, \infty), \\ (u(\cdot, 0), v(\cdot, 0))=\left(u_{0}, v_{0}\right) & \text { in } \Omega\end{cases}
$$

under the following assumptions:

(1) $\Omega$ is a bounded domain in $\mathbb{R}^{N}, N \geqslant 1$, of class $\mathcal{C}^{2}$.

(2) $\lambda, \mu \in \mathbb{R}$ and, for each $j \in\{1,2\}, \gamma_{j} \in \mathbb{R}$ and $\mathfrak{L}_{j}$ is an uniformly strongly elliptic second order differential operator in $\Omega$ of the same type as $\mathfrak{L}$.

(3) For each $j \in\{1,2\}, \mathfrak{B}_{j}:=\mathfrak{B}_{j}\left(b_{j}\right)$ stands for the boundary operator

$$
\mathfrak{B}_{j}:= \begin{cases}\mathfrak{D} & \text { on } \Gamma_{0}^{j}, \\ \partial_{\nu_{j}}+b_{j} & \text { on } \Gamma_{1}^{j},\end{cases}
$$

where $\Gamma_{0}^{j}$ and $\Gamma_{1}^{j}$ are two disjoint open and closed subsets of $\partial \Omega$ with $\Gamma_{0}^{j} \cup \Gamma_{1}^{j}=$ $\partial \Omega, b_{j} \in \mathcal{C}\left(\Gamma_{1}^{j}\right)$, and $v_{j}=\left(v_{j, 1}, \ldots, v_{j, N}\right) \in \mathcal{C}^{1}\left(\Gamma_{1}^{j} ; \mathbb{R}^{N}\right)$ is an outward pointing nowhere tangent vector field.

(4) Each of the functions $V_{j}$ and $\mathcal{X}_{j}, j \in\{1,2\}$, is non-negative measurable and bounded in $\Omega$. Moreover,

$$
\mathcal{X}_{j} \in \mathcal{A}_{\Gamma_{0}^{j}, \Gamma_{1}^{j}}^{+}(\Omega), \quad V_{j} \in \mathcal{A}_{\Gamma_{0}^{j}, \Gamma_{1}^{j}}(\Omega), \quad j \in\{1,2\} .
$$

(5) For each $j \in\{1,2\}, \Omega_{V_{j}}^{0}$ is connected and

$$
\partial \Omega_{V_{j}}^{0} \backslash \Gamma_{1}^{j} \subset \Omega, \quad \operatorname{dist}\left(\Gamma_{1}^{j}, \partial \Omega_{V_{j}}^{0} \backslash \Gamma_{1}^{j}\right)>0 .
$$


Moreover, setting

$$
\Omega_{V_{j}}^{\delta}:=\Omega_{V_{j}}^{0} \cup\left\{x \in \Omega: \operatorname{dist}\left(x, \partial \Omega_{V_{j}}^{0} \backslash \Gamma_{1}^{j}\right)<\delta\right\}
$$

we assume that there is a sequence $v_{n}^{j}, n \geqslant 1$, such that $\lim _{n \rightarrow \infty} v_{n}^{j}=0$ for which the general assumptions of Theorem 2.19(e) of Section 2 with

$$
\left(a, \Omega_{0}, \Omega_{n}\right)=\left(\mathcal{X}_{j}, \Omega_{V_{j}}^{0}, \Omega_{V_{j}}^{v_{n}^{j}}\right), \quad n \geqslant 1,
$$

is satisfied (note that $\mathcal{X}_{j} \in \mathcal{A}_{\Gamma_{0}^{j}, \Gamma_{1}^{j}}^{+}(\Omega)$ ). Furthermore, there exists $\delta_{0}>0$ such that, for each $\delta \in\left[0, \delta_{0}\right)$,

$$
\mathcal{X}_{j} \in \mathcal{A}_{\partial \Omega_{V_{j}}^{\delta} \backslash \Gamma_{1}^{j}, \partial \Omega_{V_{j}}^{\delta} \cap \Gamma_{1}^{j}}^{+}\left(\Omega_{V_{j}}^{\delta}\right), \quad j \in\{1,2\} .
$$

(6) $\left(u_{0}, v_{0}\right) \in X_{0}^{2}$ where $X_{0}:=L_{p}^{+}(\Omega)$ for some $p>\frac{N}{2}$.

Under these assumptions, for each initial data $\left(u_{0}, v_{0}\right) \in X_{0}^{2}$, (5.1) has a unique global strict solution $\left(u\left(x, t ; u_{0}, v_{0}\right), v\left(x, t ; u_{0}, v_{0}\right)\right)$ (cf. [2]). In fact, thanks to the parabolic maximum principle, for any $t>0$ we have that

$$
0 \leqslant u\left(\cdot, t ; u_{0}, v_{0}\right) \leqslant T_{1}(t) u_{0} \quad \wedge \quad 0 \leqslant v\left(\cdot, t ; u_{0}, v_{0}\right) \leqslant T_{2}(t) v_{0},
$$

where, $T_{1}(t)$ and $T_{2}(t)$ stand for the $L_{p}$-evolution operators associated with $\mathfrak{L}_{1}-\lambda$ and $\mathfrak{L}_{2}-\mu$, respectively. It is well known that most of the limiting profiles of the positive solutions of (5.1) as $t \nearrow \infty$ are given by the strong non-negative steady states of (5.1) (cf. [14] and the further developments of [11-13,24,26], and the references therein). The steady-states of (5.1) are the non-negative strong solutions of

$$
\begin{cases}\mathfrak{L}_{1} u=\lambda u-\mathcal{X}_{1}(x) u^{2}-\gamma_{1} V_{1}(x) u v & \text { in } \Omega, \\ \mathfrak{L}_{2} v=\mu v-\mathcal{X}_{2}(x) v^{2}-\gamma_{2} V_{2}(x) u v & \text { in } \Omega, \\ \mathfrak{B}_{1} u=\mathfrak{B}_{2} v=0 & \text { on } \partial \Omega .\end{cases}
$$

As we consider (5.1) to model competition between populations, we shall pay attention only to the component-wise non-negative steady states. Besides $(0,0)$, the problem (5.2) admits three types of component-wise non-negative solution couples. Namely, the solutions having one component vanishing, $(u, 0)$ or $(0, v)$, known as the semi-trivial positive solutions, and the solutions having both component positive, known as the coexistence states of (5.2). Due to Theorem 2.14, (5.2) possesses a semi-trivial positive solution of the form $(u, 0)$ if, and only if,

$$
\lambda>\sigma\left[\mathfrak{L}_{1}, \Omega, \mathfrak{B}_{1}\right] .
$$

Moreover, in this case, $\left(\Xi_{\lambda}, 0\right)$ is the unique of these semi-trivial states, where $\Xi_{\lambda}$ stands for the unique positive solution of

$$
\begin{cases}\mathfrak{L}_{1} u=\lambda u-\mathcal{X}_{1}(x) u^{2} & \text { in } \Omega, \\ \mathfrak{B}_{1} u=0 & \text { on } \partial \Omega .\end{cases}
$$


Similarly, (5.2) possesses a semi-trivial positive solution of the form $(0, v)$ if, and only if,

$$
\mu>\sigma\left[\mathfrak{L}_{2}, \Omega, \mathfrak{B}_{2}\right],
$$

and, in such case, $\left(0, \Upsilon_{\mu}\right)$ is the unique of these semi-trivial states, where $\Upsilon_{\mu}$ stands for the unique positive solution of

$$
\begin{cases}\mathfrak{L}_{2} v=\mu v-\mathcal{X}_{2}(x) v^{2} & \text { in } \Omega, \\ \mathfrak{B}_{2} v=0 & \text { on } \partial \Omega .\end{cases}
$$

The following result characterizes the stability of each of the semi-trivial positive solutions of (5.2).

Proposition 5.1. - Suppose $\lambda>\sigma\left[\mathfrak{L}_{1}, \Omega, \mathfrak{B}_{1}\right]$. Then, $\left(\Xi_{\lambda}, 0\right)$ is linearly asymptotically stable (l.a.s.) if, and only if,

$$
\mu<\sigma\left[\mathfrak{L}_{2}+\gamma_{2} V_{2} \Xi_{\lambda}, \Omega, \mathfrak{B}_{2}\right]
$$

linearly neutrally stable (l.n.s.) if, and only if,

$$
\mu=\sigma\left[\mathfrak{L}_{2}+\gamma_{2} V_{2} \boldsymbol{\Xi}_{\lambda}, \Omega, \mathfrak{B}_{2}\right],
$$

and linearly unstable (l.u.) if, and only if,

$$
\mu>\sigma\left[\mathfrak{L}_{2}+\gamma_{2} V_{2} \boldsymbol{\Xi}_{\lambda}, \Omega, \mathfrak{B}_{2}\right] .
$$

By symmetry, in case $\mu>\sigma\left[\mathfrak{L}_{2}, \Omega, \mathfrak{B}_{2}\right]$, the state $\left(0, \Upsilon_{\mu}\right)$ is l.a.s. if $\lambda<\sigma\left[\mathfrak{L}_{1}+\gamma_{1} V_{1} \Upsilon_{\mu}\right.$, $\left.\Omega, \mathfrak{B}_{1}\right]$, l.n.s. if $\lambda=\sigma\left[\mathfrak{L}_{1}+\gamma_{1} V_{1} \Upsilon_{\mu}, \Omega, \mathfrak{B}_{1}\right]$ and l.u. if $\lambda>\sigma\left[\mathfrak{L}_{1}+\gamma_{1} V_{1} \Upsilon_{\mu}, \Omega, \mathfrak{B}_{1}\right]$.

Proof. - Suppose $\lambda>\sigma\left[\mathfrak{L}_{1}, \Omega, \mathfrak{B}_{1}\right]$. By definition, the linear stability of $\left(\Xi_{\lambda}, 0\right)$ is given by the sign of the real parts of the eigenvalues of the linearizations of (5.2) at $\left(\Xi_{\lambda}, 0\right)$, i.e., by the signs of the real parts of the $\tau$ 's for which the following linear problem possesses a solution $(u, v) \neq(0,0)$ :

$$
\begin{cases}\mathfrak{L}_{1} u=\left(\lambda-2 \mathcal{X}_{1} \Xi_{\lambda}\right) u-\gamma_{1} V_{1} \Xi_{\lambda} v+\tau u & \text { in } \Omega, \\ \mathfrak{L}_{2} v=\left(\mu-\gamma_{2} V_{2} \Xi_{\lambda}\right) v+\tau v & \text { in } \Omega, \\ \mathfrak{B}_{1} u=\mathfrak{B}_{2} v=0 & \text { on } \partial \Omega .\end{cases}
$$

If $v=0$, then (5.5) becomes

$$
\begin{cases}\mathfrak{L}_{1} u=\left(\lambda-2 \mathcal{X}_{1} \Xi_{\lambda}\right) u+\tau u & \text { in } \Omega \\ \mathfrak{B}_{1} u=0 & \text { on } \partial \Omega\end{cases}
$$

Moreover, thanks to Proposition 2.5 and Lemma 2.11, we have that

$$
\sigma\left[\mathfrak{L}_{1}+2 \mathcal{X}_{1} \Xi_{\lambda}-\lambda, \Omega, \mathfrak{B}_{1}\right]>\sigma\left[\mathfrak{L}_{1}+\mathcal{X}_{1} \Xi_{\lambda}-\lambda, \Omega, \mathfrak{B}_{1}\right]=0 .
$$


Thus, since the principal eigenvalue is dominant, we find that

$$
\operatorname{Re} \tau>\sigma\left[\mathfrak{L}_{1}+2 \mathcal{X}_{1} \Xi_{\lambda}-\lambda, \Omega, \mathfrak{B}_{1}\right]>0
$$

and, therefore, the linear stability of $\left(\Xi_{\lambda}, 0\right)$ is determined from the signs of the eigenvalues of (5.5) possessing an eigenfunction of the form $(u, v)$ with $v \neq 0$, i.e., by the sign of

$$
\delta_{\mu}:=\mu-\sigma\left[\mathfrak{L}_{2}+\gamma_{2} V_{2} \Xi_{\lambda}, \Omega, \mathfrak{B}_{2}\right]=-\sigma\left[\mathfrak{L}_{2}+\gamma_{2} V_{2} \Xi_{\lambda}-\mu, \Omega, \mathfrak{B}_{2}\right] .
$$

Indeed, if $\delta_{\mu}<0$, then

$$
-\delta_{\mu}=\sigma\left[\mathfrak{L}_{2}+\gamma_{2} V_{2} \Xi_{\lambda}-\mu, \Omega, \mathfrak{B}_{2}\right]>0
$$

and, since the principal eigenvalue is dominant, any eigenvalue of (5.5) satisfies

$$
\operatorname{Re} \tau \geqslant \sigma\left[\mathfrak{L}_{2}+\gamma_{2} V_{2} \Xi_{\lambda}-\mu, \Omega, \mathfrak{B}_{2}\right]>0 .
$$

Therefore, in this case $\left(\Xi_{\lambda}, 0\right)$ is linearly asymptotically stable.

Now, suppose $\delta_{\mu}=0$. Then,

$$
-\delta_{\mu}=\sigma\left[\mathfrak{L}_{2}+\gamma_{2} V_{2} \Xi_{\lambda}-\mu, \Omega, \mathfrak{B}_{2}\right]=0
$$

and, hence, the pair $(\tau, v)=\left(0, \varphi_{v}\right)$, where $\varphi_{v}>0$ is the principal eigenfunction associated with $\left(\mathfrak{L}_{2}+\gamma_{2} V_{2} \Xi_{\lambda}-\mu, \Omega, \mathfrak{B}_{2}\right)$, solves the $v$-equation of (5.5). Moreover, thanks to (5.7), the $u$-equation of (5.5) possesses a unique solution - in $u$ - for $(\tau, v)=\left(0, \varphi_{v}\right)$. Namely,

$$
u=-\gamma_{1}\left(\mathfrak{L}_{1}+2 \mathcal{X}_{1} \Xi_{\lambda}-\lambda\right)^{-1}\left(V_{1} \Xi_{\lambda} \varphi_{v}\right) .
$$

Thus, $\tau=0$ is an eigenvalue of (5.5). As any other value of $\tau$ for which the $v$-equation of (5.5) can be solved must be positive, we obtain that $\left(\Xi_{\lambda}, 0\right)$ is linearly neutrally stable.

Finally, suppose $\delta_{\mu}>0$. Then, adapting the argument of the previous case, one readily gets that $-\delta_{\mu}<0$ is an eigenvalue of (5.5) and, therefore, $\left(\Xi_{\lambda}, 0\right)$ is linearly unstable.

By symmetry, one obtains the corresponding characterizations for $\left(0, \Upsilon_{\mu}\right)$.

Thanks to the linearized stability principle, $\left(\Xi_{\lambda}, 0\right)$ (resp. $\left(0, \Upsilon_{\mu}\right)$ ) is exponentially asymptotically stable if it is linearly asymptotically stable, and it is unstable if it is linearly unstable - as steady states of (5.1). The following concept is very important in mathematical biology. Subsequently, $P_{C_{0}^{1}(\bar{\Omega})}$ stands for the cone of positive functions of $C_{0}^{1}(\bar{\Omega})$.

DEFINITION 5.2. - The problem (5.1) is permanent - or, equivalently, compressive - if there is a subdomain

$$
\mathfrak{R} \subset\left(\operatorname{Int} P_{C_{0}^{1}(\bar{\Omega})}\right)^{2}
$$

such that

$$
\left(u\left(\cdot, t ; u_{0}, v_{0}\right), v\left(\cdot, t ; u_{0}, v_{0}\right)\right) \in \Re
$$


for each $\left(u_{0}, v_{0}\right) \in X_{0}^{2}, u_{0}>0, v_{0}>0$, after some time $t_{0}:=t\left(u_{0}, v_{0}\right)$.

The abstract theory developed in [13] and the results of [10] and [19] are easily adapted to show that global extinction of some of the species occurs if (5.2) does not admit a coexistence state (cf. [15] for a further general version of that result).

Thus, (5.2) must possess a coexistence state if (5.1) is permanent. Let $\left(u_{0}, v_{0}\right)$ be a coexistence state of (5.2). Then, thanks to Proposition 2.5, we have

$$
\sigma\left[\mathfrak{L}_{1}-\lambda, \Omega, \mathfrak{B}_{1}\right]<\sigma\left[\mathfrak{L}_{1}-\lambda+\mathcal{X}_{1} u_{0}+\gamma_{1} V_{1} v_{0}, \Omega, \mathfrak{B}_{1}\right]=0
$$

and

$$
\sigma\left[\mathfrak{L}_{2}-\mu, \Omega, \mathfrak{B}_{2}\right]<\sigma\left[\mathfrak{L}_{2}-\mu+\mathcal{X}_{2} v_{0}+\gamma_{2} V_{2} u_{0}, \Omega, \mathfrak{B}_{2}\right]=0 .
$$

Thus,

$$
\lambda>\sigma\left[\mathfrak{L}_{1}, \Omega, \mathfrak{B}_{1}\right] \quad \wedge \quad \mu>\sigma\left[\mathfrak{L}_{2}, \Omega, \mathfrak{B}_{2}\right] .
$$

In particular, (5.2) exhibits the two possible semi-trivial positive solutions, $\left(\Xi_{\lambda}, 0\right)$ and $\left(0, \Upsilon_{\mu}\right)$. Obviously, (5.1) cannot be permanent if some of these semi-trivial states is linearly asymptotically stable. Therefore, thanks to Proposition 5.1, the following estimates are necessary for permanence

$$
\lambda \geqslant \sigma\left[\mathfrak{L}_{1}+\gamma_{1} V_{1} \Upsilon_{\mu}, \Omega, \mathfrak{B}_{1}\right] \wedge \mu \geqslant \sigma\left[\mathfrak{L}_{2}+\gamma_{2} V_{2} \Xi_{\lambda}, \Omega, \mathfrak{B}_{2}\right] .
$$

Conversely, the following result is satisfied (cf. $[13,16]$ and [8] for some previous results in this direction).

THEOREM 5.3. - Suppose (5.8) and $\left(\Xi_{\lambda}, 0\right),\left(0, \Upsilon_{\mu}\right)$ are linearly unstable, i.e., thanks to Proposition 5.1,

$$
\lambda>\sigma\left[\mathfrak{L}_{1}+\gamma_{1} V_{1} \Upsilon_{\mu}, \Omega, \mathfrak{B}_{1}\right] \wedge \mu>\sigma\left[\mathfrak{L}_{2}+\gamma_{2} V_{2} \Xi_{\lambda}, \Omega, \mathfrak{B}_{2}\right] .
$$

Then, (5.1) possesses a coexistence state and it is compressive.

Proof. - We shall use a practical persistence argument based upon the arguments of [16] and [9]. The existence of the coexistence state can be obtained by using the theory of [13]. The permanence can be obtained arguing as follows. Pick $u_{0}, v_{0} \in X_{0}$ such that $u_{0}>0$ and $v_{0}>0$. Then, thanks to the parabolic maximum principle,

$$
\begin{aligned}
& 0<u\left(\cdot, t ; u_{0}, v_{0}\right)<\Phi_{\left[\mathfrak{L}_{1}, \lambda, \mathcal{X}_{1}, \mathfrak{B}_{1}\right]}\left(\cdot, t ; u_{0}\right), \\
& 0<v\left(\cdot, t ; u_{0}, v_{0}\right)<\Phi_{\left[\mathfrak{L}_{2}, \mu, \mathcal{X}_{2}, \mathfrak{B}_{2}\right]}\left(\cdot, t ; v_{0}\right),
\end{aligned}
$$

where $\Phi_{[\mathfrak{L}, \gamma, \mathcal{X}, \mathfrak{B}]}\left(x, t ; w_{0}\right)$ stands for the unique solution of the parabolic problem

$$
\begin{cases}\frac{\partial w}{\partial t}+\mathfrak{L} w=\gamma w-\mathcal{X} w^{2} & \text { in } \Omega \times(0, \infty), \\ \mathfrak{B} w=0 & \text { on } \partial \Omega \times(0, \infty), \\ w(\cdot, 0)=w_{0} & \text { in } \Omega .\end{cases}
$$


On the other hand, thanks to Theorem 2.14, we have

$$
\begin{aligned}
& \lim _{t>\infty}\left\|\Phi_{\left[\mathfrak{L}_{1}, \lambda, \mathcal{X}_{1}, \mathfrak{B}_{1}\right]}\left(\cdot, t ; u_{0}\right)-\Xi_{\lambda}\right\|_{C_{0}^{1}(\bar{\Omega})}=0, \\
& \lim _{t>\infty}\left\|\Phi_{\left[\mathfrak{L}_{2}, \mu, \mathcal{X}_{2}, \mathfrak{B}_{2}\right]}\left(\cdot, t ; v_{0}\right)-\Upsilon_{\mu}\right\|_{C_{0}^{1}(\bar{\Omega})}=0,
\end{aligned}
$$

and, hence, for any $\varepsilon>0$ there exists $t_{\varepsilon}>0$ such that

$$
\left\{\begin{array}{l}
0<u\left(\cdot, t ; u_{0}, v_{0}\right)<\Xi_{\lambda}+\varepsilon, \\
0<v\left(\cdot, t ; u_{0}, v_{0}\right)<\Upsilon_{\mu}+\varepsilon
\end{array} \quad \text { in } \Omega \text { for each } t \geqslant t_{\varepsilon} .\right.
$$

Choose $\varepsilon>0$ sufficiently small so that

$$
\lambda>\sigma\left[\mathfrak{L}_{1}+\gamma_{1} V_{1}\left(\Upsilon_{\mu}+\varepsilon\right), \Omega, \mathfrak{B}_{1}\right] \wedge \mu>\sigma\left[\mathfrak{L}_{2}+\gamma_{2} V_{2}\left(\Xi_{\lambda}+\varepsilon\right), \Omega, \mathfrak{B}_{2}\right] .
$$

Substituting the second estimate of (5.12) into the $u$-equation of (5.1), gives

$$
\frac{\partial u}{\partial t}+\mathfrak{L}_{1} u=\lambda u-\mathcal{X}_{1} u^{2}-\gamma_{1} V_{1} u v>\lambda u-\mathcal{X}_{1} u^{2}-\gamma_{1} V_{1}\left(\Upsilon_{\mu}+\varepsilon\right) u
$$

for each $t \geqslant t_{\varepsilon}$, and, hence, thanks to the parabolic maximum principle,

$$
u\left(\cdot, t ; u_{0}, v_{0}\right) \geqslant \Phi_{\left[\mathfrak{L}_{1}+\gamma_{1} V_{1}\left(\Upsilon_{\mu}+\varepsilon\right), \lambda, \mathcal{X}_{1}, \mathfrak{B}_{1}\right]}\left(\cdot, t ; u\left(\cdot, t_{\varepsilon} ; u_{0}, v_{0}\right)\right) \quad \text { in } \Omega \text { for each } t \geqslant t_{\varepsilon} .
$$

On the other hand, thanks to Theorem 2.14,

$$
\lim _{t>\infty} \Phi_{\left[\mathfrak{L}_{1}+\gamma_{1} V_{1}\left(\Upsilon_{\mu}+\varepsilon\right), \lambda, \mathcal{X}_{1}, \mathfrak{B}_{1}\right]}\left(\cdot, t ; u\left(\cdot, t_{\varepsilon} ; u_{0}, v_{0}\right)\right)=u_{\left[\mathfrak{L}_{1}+\gamma_{1} V_{1}\left(\Upsilon_{\mu}+\varepsilon\right), \lambda, \mathcal{X}_{1}, \Omega, \mathfrak{B}_{1}\right]}
$$

uniformly in $\bar{\Omega}$, where $u_{\left[\mathfrak{L}_{1}+\gamma_{1} V_{1}\left(\Upsilon_{\mu}+\varepsilon\right), \lambda, \mathcal{X}_{1}, \Omega, \mathfrak{B}_{1}\right]}$ stands for the unique positive solution of

$$
\begin{cases}{\left[\mathfrak{L}_{1}+\gamma_{1} V_{1}\left(\Upsilon_{\mu}+\varepsilon\right)\right] u=\lambda u-\mathcal{X}_{1} u^{2}} & \text { in } \Omega, \\ \mathfrak{B}_{1} u=0 & \text { on } \partial \Omega\end{cases}
$$

whose existence is guaranteed from the first inequality of (5.13). Thus, for each $\varepsilon>0$ sufficiently small

$$
\liminf _{t \nearrow \infty} u\left(\cdot, t ; u_{0}, v_{0}\right) \geqslant u_{\left[\mathfrak{L}_{1}+\gamma_{1} V_{1}\left(\Upsilon_{\mu}+\varepsilon\right), \lambda, \mathcal{X}_{1}, \Omega, \mathfrak{B}_{1}\right]}
$$

and, therefore,

$$
\liminf _{t \nearrow \infty} u\left(\cdot, t ; u_{0}, v_{0}\right) \geqslant u_{\left[\mathfrak{L}_{1}+\gamma_{1} V_{1} \Upsilon_{\mu}, \lambda, \mathcal{X}_{1}, \Omega ; \mathfrak{B}_{1}\right]} .
$$

Similarly,

$$
\liminf _{t \nearrow \infty} v\left(\cdot, t ; u_{0}, v_{0}\right) \geqslant v_{\left[\mathfrak{L}_{2}+\gamma_{2} V_{2} \Xi_{\lambda}, \mu, \mathcal{X}_{2}, \Omega, \mathfrak{B}_{2}\right]},
$$

where $v_{\left[\mathfrak{L}_{2}+\gamma_{2} V_{2} \Xi_{\lambda}, \mu, \mathcal{X}_{2}, \Omega, \mathfrak{B}_{2}\right]}$ stands for the unique positive solution of

$$
\begin{cases}{\left[\mathfrak{L}_{2}+\gamma_{2} V_{2} \Xi_{\lambda}\right] v=\mu v-\mathcal{X}_{2} v^{2}} & \text { in } \Omega \\ \mathfrak{B}_{2} v=0 & \text { on } \partial \Omega\end{cases}
$$


S. CANO-CASANOVA, J. LÓPEZ-GÓMEZ / Ann. I. H. Poincaré - AN 20 (2003) 999-1041 1039

This completes the proof of the theorem.

Now, combining Theorem 5.3 together with Proposition 2.4 and Theorem 2.9, we obtain the following result.

COROLlary 5.4. - Suppose that

$$
\lambda>\sigma\left[\mathfrak{L}_{1}, \Omega_{V_{1}}^{0}, \mathfrak{B}_{1}\left(b_{1}, \Omega_{V_{1}}^{0}\right)\right] \wedge \mu>\sigma\left[\mathfrak{L}_{2}+\gamma_{2} V_{2} \Xi_{\lambda}, \Omega, \mathfrak{B}_{2}\right] .
$$

Then, (5.1) possesses a coexistence state and it is permanent for any $\gamma_{1} \geqslant 0$. Similarly, if

$$
\lambda>\sigma\left[\mathfrak{L}_{1}+\gamma_{1} V_{1} \Upsilon_{\mu}, \Omega, \mathfrak{B}_{1}\right] \wedge \mu>\sigma\left[\mathfrak{L}_{2}, \Omega_{V_{2}}^{0}, \mathfrak{B}_{2}\left(b_{2}, \Omega_{V_{2}}^{0}\right)\right],
$$

then (5.1) possesses a coexistence state and it is permanent for any $\gamma_{2} \geqslant 0$.

It should be noted that Corollary 5.4 is optimal. Moreover, thanks to Theorem 1.3 we obtain the following

THEOREM 5.5. - Suppose (2.8) on $\Gamma_{1}^{1} \cap \partial \Omega_{V_{1}}^{0}$ and (5.14), fix $\gamma_{2}$ and regard to $\gamma_{1}$ as a parameter. Then, the $u$-component of any coexistence state $(u, v)=\left(u\left(\gamma_{1}\right), v\left(\gamma_{1}\right)\right)$ of (5.2) must satisfy

$$
\lim _{\gamma_{1} \nearrow \infty} u\left(\gamma_{1}\right)= \begin{cases}u_{\left[\mathfrak{L}_{1}, \lambda, \mathcal{X}_{1}, \Omega_{V_{1}}^{0}, \mathfrak{B}_{1}\left(b_{1}, \Omega_{V_{1}}^{0}\right)\right]} & \text { in } \Omega_{V_{1}}^{0}, \\ 0 & \text { in } \Omega \backslash \Omega_{V_{1}}^{0} .\end{cases}
$$

Similarly, when (5.15) is satisfied and (2.8) holds on $\Gamma_{1}^{2} \cap \partial \Omega_{V_{2}}^{0}$, fixing $\gamma_{1}$ and regarding to $\gamma_{2}$ as a parameter gives

$$
\lim _{\gamma_{2} \nearrow \infty} v\left(\gamma_{2}\right)= \begin{cases}v_{\left[\mathfrak{L}_{2}, \mu, \mathcal{X}_{2}, \Omega_{V_{2}}^{0}, \mathfrak{B}_{2}\left(b_{2}, \Omega_{V_{2}}^{0}\right)\right]} & \text { in } \Omega_{V_{2}}^{0}, \\ 0 & \text { in } \Omega \backslash \Omega_{V_{2}}^{0} .\end{cases}
$$

These convergences must be understood in the sense of Theorem 1.3.

Proof. - Suppose (5.14) and let $(u, v)$ be a coexistence state of (5.2). Then, thanks to Theorem 2.16 , it is easily shown that

$$
u_{\left[\mathfrak{L}_{1}+\gamma_{1} V_{1} \Upsilon_{\mu}, \lambda, \mathcal{X}_{1}, \Omega, \mathfrak{B}_{1}\right]}<u<u_{\left[\mathfrak{L}_{1}, \lambda, \mathcal{X}_{1}, \Omega, \mathfrak{B}_{1}\right]}
$$

and

$$
v_{\left[\mathfrak{L}_{2}+\gamma_{2} V_{2} \Xi_{\lambda}, \mu, \mathcal{X}_{2}, \Omega, \mathfrak{B}_{2}\right]}<v<v_{\left[\mathfrak{L}_{2}, \mu, \mathcal{X}_{2}, \Omega, \mathfrak{B}_{2}\right]} .
$$

Therefore, thanks again to Theorem 2.16, we have

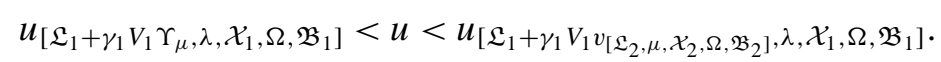

Thanks to Theorem 1.3, passing to the limit as $\gamma_{1} \nearrow \infty$ completes the proof of (5.16). The same argument can be easily adapted to prove the validity of (5.17) under condition (5.15). 
1040 S. CANO-CASANOVA, J. LÓPEZ-GÓMEZ / Ann. I. H. Poincaré - AN 20 (2003) 999-1041

\section{Acknowledgements}

Part of this work has been supported by the Spanish Ministry of Science and Technology under grant BFM2000-0797.

\section{REFERENCES}

[1] H. Amann, Dual semigroups and second order linear elliptic boundary value problems, Israel J. Math. 45 (1983) 225-254.

[2] H. Amann, Linear and Quasilinear Parabolic Problems, in: Monographs Math., Vol. 89, Birkhäuser, Basel, 1995.

[3] H. Amann, J. López-Gómez, A priori bounds and multiple solutions for superlinear indefinite elliptic problems, J. Differential Equations 146 (1998) 336-374.

[4] M. Begon, J.L. Harper, C.R. Townsend, Ecology, Individual, Populations and Communities, Blackwell Scientific Publications, Cambridge, MA, 1990.

[5] S. Cano-Casanova, Existence and structure of the set of positive solutions of a general class of sublinear elliptic non-classical mixed boundary value problems, Nonlinear Anal. 49 (2002) 361-430.

[6] S. Cano-Casanova, J. López-Gómez, Properties of the principal eigenvalues of a general class of non-classical mixed boundary value problems, J. Differential Equations 178 (2002) 123-211.

[7] S. Cano-Casanova, J. López-Gómez, Varying domains in a general class of sublinear elliptic problems, submited.

[8] R.S. Cantrell, C. Cosner, On the effects of spatial heterogeneity on the persistence of interacting species, J. Math. Biol. 37 (1998) 103-145.

[9] R.S. Cantrell, C. Cosner, V. Hutson, Permanence in some diffusive Lotka-Volterra models for three interacting species, Dynamic Systems Appl. 2 (1993) 505-530.

[10] E.N. Dancer, On the existence and uniqueness of positive solutions for competing species models with diffusion, Trans. Amer. Math. Soc. 326 (1991) 829-859.

[11] E.N. Dancer, Positivity of maps and applications, in: M. Matzeu, A. Vignoli (Eds.), Topological Nonlinear Analysis, Degree, Singularities and Variations, in: Progr. Nonlinear Differential Equations Appl., Vol. 15, Birkhäuser, Basel, 1995, pp. 303-340.

[12] P. Hess, Periodic-Parabolic Boundary Value Problems and Positivity, Longman, Harlow, 1991.

[13] P. Hess, A.C. Lazer, On an abstract competition model and applications, Nonlinear Anal. 16 (1991) 917-940.

[14] M. Hirsch, Stability and convergence in strongly monotone dynamical systems, J. Reine Angew. Math. 383 (1988) 1-58.

[15] S.B. Hsu, H.L. Smith, P. Waltman, Competitive exclusion and coexistence for competitive systems on ordered Banach spaces, Trans. Amer. Math. Soc. 348 (1996) 4083-4094.

[16] J. López-Gómez, Permanence under strong competition, in: World Sci. Ser. Appl. Anal., Vol. 4, Word Sci. Publishing, River Edge, NJ, 1995, 473-488.

[17] J. López-Gómez, The maximum principle and the existence of principal eigenvalues for some linear weighted boundary value problems, J. Differential Equations 127 (1996) 263294.

[18] J. López-Gómez, M. Molina-Meyer, The maximum principle for cooperative weakly coupled elliptic systems and some applications, Differential Integral Equations 7 (1994) 383-398. 
S. CANO-CASANOVA, J. LÓPEZ-GÓMEZ / Ann. I. H. Poincaré - AN 20 (2003) 999-1041 1041

[19] J. López-Gómez, J.C. Sabina, Coexistence states and global attractivity for some convective diffusive competition models, Trans. Amer. Math. Soc. 347 (1995) 3797-3833.

[20] J.D. Murray, Mathematical Biology, in: Biomathematics Texts, Vol. 19, Springer, Berlin, 1989.

[21] J. Neças, Les méthodes directes en théorie des équations elliptiques, Academia, Prague, 1967.

[22] A. Okubo, Diffusion and Ecological Problems: Mathematical Models, Springer, Berlin, 1980.

[23] H.H. Schaefer, Topological Vector Spaces, Springer, New York, 1971.

[24] H.L. Smith, H.R. Thieme, Stable coexistence and bio-stability for competitive systems of ordered banach spaces, J. Differential Equations 176 (2001) 195-222.

[25] E.M. Stein, Singular Integrals and Differentiability Properties of Functions, Princeton University Press, Princeton, NJ, 1970.

[26] P. Takác, Discrete monotone dynamics and time periodic competition between two species, Differential Integral Equations 10 (1997) 547-576.

[27] E.O. Wilson, Sociobiology, Harvard University Press, Cambridge, MA, 1980. 